Distribution, abundance and threats to populations of

Baird's tapir (Tapirus bairdii) and white-lipped peccary

(Tayassu peccary) populations in Mexico

\title{
Distribución, abundancia y amenazas a las poblaciones de tapir centroamericano (Tapirus bairdii) y pecarí de labios blancos (Tayassu pecari) en México
}

\author{
Eduardo J. Naranjo ${ }^{1 *}$, Saúl A. Amador-Alcalá ${ }^{1}$, Fredy A. Falconi-Briones ${ }^{1}$ y Rafael A. Reyna-Hurtado ${ }^{2}$ \\ ${ }^{1}$ El Colegio de la Frontera Sur. Carretera Panamericana y Periférico Sur s/n, San Cristóbal de Las Casas, Chiapas 29290, México. Email: \\ enaranjo@ecosur.mx (EJN), sulocarioca@yahoo.com.mx (SAAA), falconi.fab@hotmail.com (AFB). \\ ${ }^{2}$ Rafael A. Reyna-Hurtado; El Colegio de la Frontera Sur. Av. Rancho Polígono 2-A, Ciudad Industrial Lerma, Campeche, Campeche \\ 24500, México. Email: rreyna@ecosur.mx (RARH). \\ ${ }^{*}$ Corresponding author
}

Introduction: Baird's tapir (Tapirus bairdii) and the white-lipped peccary (Tayassu pecari) play important roles in the dynamics of tropical forests where they live through herbivory, seed dispersal, and seed predation. These mammals have constituted relevant food sources for residents of rural communities of Mesoamerica. Both ungulates are listed as endangered species in Mexico's Official Norm 059 (NOM-059-SEMARNAT-2010). Their main threats are habitat loss and poaching throughout their distribution range in Mexico. The central purpose of this study was to assess the population status of Baird's tapir and the white-lipped peccary in Mexico, proposing a strategy for their conservation.

Methods: From November 2010 through November 2012 we did rapid assessments of presence, relative abundance, and threats for Baird's tapir and white-lipped peccary populations in ten localities across five states of southeastern Mexico. We used three complementary techniques: line transect sampling, camera-trapping, and interviews with inhabitants of rural communities. We analyzed our own data and available information on both focal species in Mexico for generating proposals confirming their status as endangered in the Official Norm 059 by means of the Risk Assessment Method (MER, for its Spanish initials), required by the Ministry of Environment and Natural Resources.

Results and discussion: Current verified distribution areas of the white-lipped peccary are located in the states of Campeche, Chiapas, Oaxaca, Quintana Roo, Veracruz, and Yucatan. Baird's tapir is present in even more localities within the same states excluding Yucatan. Because of their extensive area and good habitat quality, the critical locations for conserving both species in the country are: Calakmul-Balamkú-Balam-Kin (Campeche), Montes AzulesLacantún (Chiapas), Los Chimalapas (Oaxaca), and Sian Ka'an-forest ejidos of central Quintana Roo. The highest estimates of tapir abundance were recorded in La Fraylescana, Uxpanapa, and Los Chimalapas (sightings and tracks), as well as in Uxpanapa, Selva Lacandona, and Balam-kin (photographs). The highest white-lipped peccary abundances were found in Sian Ka'an and Calakmul (sightings and tracks), as well as in Balam-kin and Calakmul (photographs).

The main threats for conserving both ungulate species in Mexico are deforestation and habitat fragmentation, unregulated hunting, forest fires, and disease transmitted by livestock. Unexpected recent records of the two species in specific localities of Campeche, Veracruz, and Yucatan encourage the continuity and widening of research on the distribution and conservation status of these endangered mammals in southern and southeastern Mexico.

Key words: Abundance, conservation, distribution, Mexico, Tapirus bairdii, Tayassu pecari, threats. 


\section{Introducción}

La conservación y el uso sustentable de especies de fauna silvestre requieren necesariamente de información básica sobre la distribución, abundancia y biología de las poblaciones de interés (Caughley y Sinclair 1994; Ojasti y Dallmeier 2000). Esta clase de información es particularmente importante cuando las especies de interés se encuentran amenazadas o en peligro de extinción debido a la pérdida de hábitat y a la cacería sin control, como es el caso de numerosos vertebrados neotropicales, entre los que se encuentran el tapir centroamericano (Tapirus bairdii) y el pecarí de labios blancos (Tayassu pecari). Además de representar recursos alimentarios importante para los pobladores de comunidades rurales de Mesoamérica, estos dos mamíferos juegan un papel importante en la dinámica de los bosques tropicales donde habitan a través de los procesos de herbivoría, dispersión y depredación de semillas de numerosas especies vegetales (Bodmer 1991; March 1993; Olmos 1997).

En México, el tapir y el pecarí de labios blancos están considerados en peligro de extinción (SEMARNAT 2010), y ambas se encuentran en la lista de especies prioritarias de la Comisión Nacional de Areas Naturales Protegidas (CONANP 2009). La distribución histórica de ambas especies en México incluía gran parte de los bosques tropicales y mesófilos del sur. Sin embargo, en la actualidad estos ungulados se encuentran solamente en algunas áreas silvestres extensas remanentes, principalmente dentro de áreas protegidas (March 2005; March y Naranjo 2005). Aunque el estado de las poblaciones de las dos especies se conoce para algunas áreas de Chiapas, Campeche y Oaxaca, parece evidente que las tendencias apuntan hacia la declinación y el aislamiento ante el avance de la actividad humana en las áreas tropicales del país (Naranjo 2009; Naranjo 2014; Reyna 2007; Reyna et al. 2014). De aquí se desprende la importancia de generar información que ofrezca un panorama más completo y actualizado sobre la situación de ambas especies en apoyo a las estrategias y políticas que requieren aplicarse para evitar su extinción en México. A continuación se presenta una síntesis del conocimiento sobre el estado de las poblaciones de cada especie en el país:

El tapir en México. El tapir centroamericano (Figura 1) se encuentra en peligro de extinción tanto a nivel nacional (SEMARNAT 2010) como internacional (IUCN 2012), y es considerado especie prioritaria por la CONANP (2009). La información disponible para el tapir en el subsistema de especies en riesgo y prioritarias del Sistema Nacional de Información Biológica (SNIB) que administra la Comisión Nacional para el Conocimiento y Uso de la Biodiversidad (CONABIO) es deficiente y no está actualizada (Gómez-Nísino 2006). Sin embargo, dos trabajos publicados en los últimos años (Naranjo 2009; Naranjo 2014) resumen el estado del conocimiento de esta especie en México de una manera más completa. La distribución histórica de este ungulado incluyó gran parte de las áreas tropicales húmedas del sur y sureste del país (March 1994). Sin embargo, en la actualidad esta especie solamente está presente en algunas áreas silvestres extensas en los estados de Campeche, Chiapas, Oaxaca, Quintana Roo, Veracruz, y posiblemente Yucatán y Tabasco (Botello et al. 2014; Mendoza et al. 2013; Naranjo et al. 2013). Las regiones con presencia verificada de tapires son: Reserva de la Biósfera Calakmul y sureste de Campeche; Reserva de la Biósfera Sian Ka'an, centro y sur de Quintana Roo; Reservas de la Biósfera El Ocote, El Triunfo, La Sepultura, Lacantún y Montes Azules, Selva Lacandona y Sierra Madre de Chiapas; Los Chimalapas, Parque Nacional Lagunas de Chacahua y Sierra Mixe, Oaxaca; y Uxpanapa, Veracruz (Botello et al. 2014; Mendoza et al. 2013; Naranjo et al. 2013). Aunque no existen registros verificados recientes, es probable que aún existan pequeñas poblaciones aisladas de tapires en la Reserva de la Biósfera Pantanos de Centla y zona fronteriza de Tabasco, y en el extremo sur del estado de Yucatán (Naranjo 2009; Naranjo et al. 2013). 
La investigación realizada sobre el tapir en el país se ha limitado a unas pocas poblaciones. La abundancia poblacional, las preferencias de hábitat, el rango de acción individual y los hábitos de alimentación de tapires han sido estudiados en algunas localidades de Chiapas Campeche y Oaxaca mediante conteos de individuos y rastros en transectos lineales (Lira y Naranjo 2005; Muench 2001; Naranjo y Cruz 1998; Naranjo y Bodmer 2002), observación de plantas ramoneadas y análisis de excrementos (Cruz 2001; Naranjo y Cruz 1998; O'Farril et al. 2006; Rivadeneyra 2007; Naranjo 2009), radiotelemetría (Lira 2006; Naranjo y Bodmer 2002) y foto-trampeo (CarbajalBorges et al. 2014; Lira et al. 2014; Pérez y Matus 2010). Las estimaciones de densidad disponibles para México se ubican entre 0.01 y 0.5 tapires $/ \mathrm{km}^{2}$ (Carbajal-Borges et al. 2014; Naranjo 2009). Considerando estas densidades tan bajas, resulta evidente que solo unas pocas reservas en el país parecen ser suficientemente grandes para albergar poblaciones viables de tapires conformadas por varios cientos de individuos (Naranjo 2009). Los factores de amenaza más importantes para el tapir son indudablemente la pérdida de hábitat y la cacería sin control (March y Naranjo 2005; Naranjo 2014).

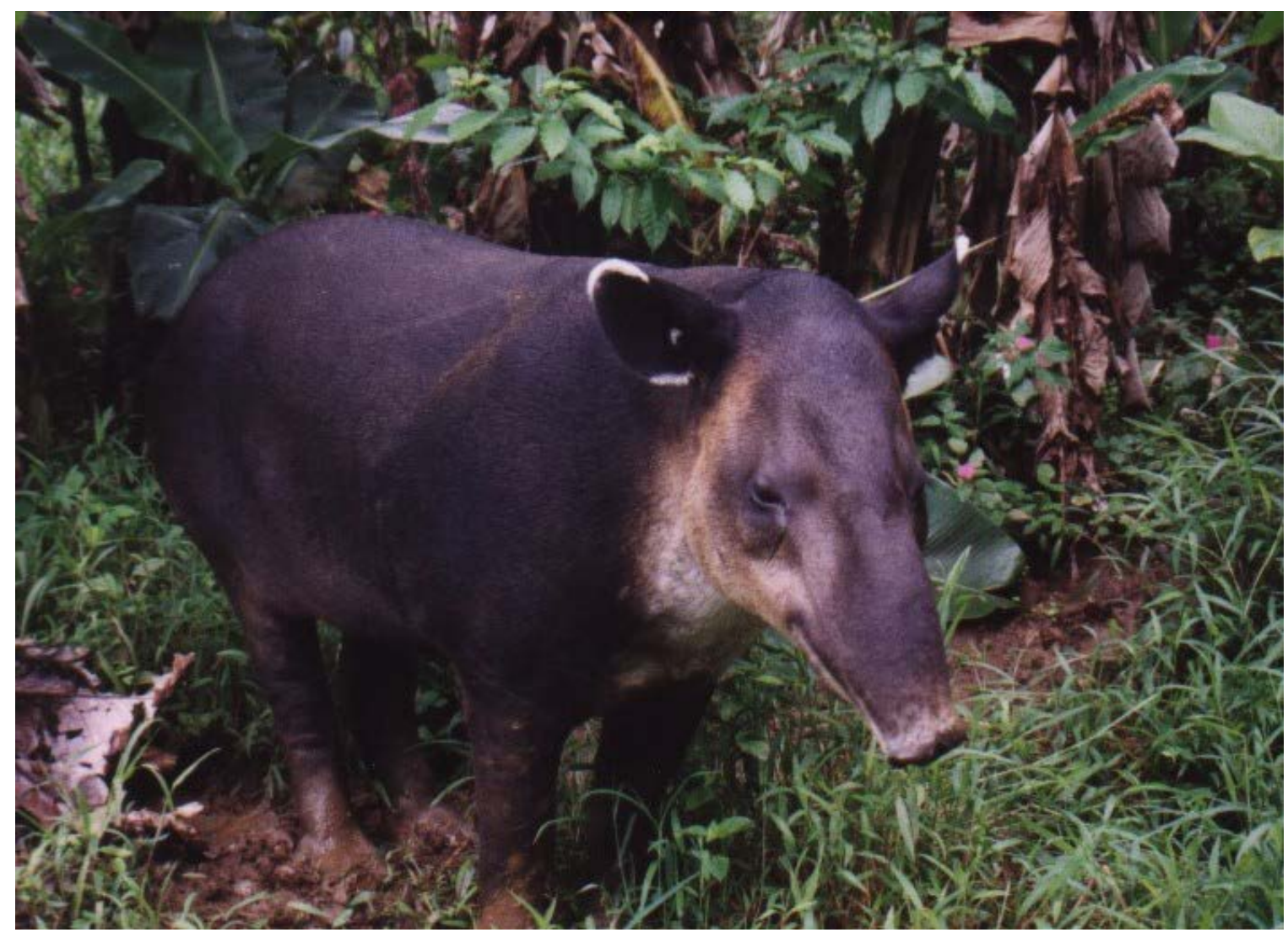

Figura 1. Tapir centroamericano (Tapirus bairdii). Selva Lacandona, Chiapas. Foto: Eduardo Naranjo.

El pecarí de labios blancos en México. El pecarí de labios blancos (Figura 2) ha sido escasamente estudiado en México, y a solicitud (mediante el procedimiento MER) de dos de los autores del presente estudio (EJN y RRH), ha sido incluido recientemente como especie en peligro de extinción en la NOM-059-2010 (SEMARNAT 2010). Este pecarí además figura en la lista de especies prioritarias de la CONANP (2009). La distribución histórica de este mamífero en el país incluyó las planicies costeras cubiertas por bosques tropicales húmedos y subhúmedos del Golfo de México, Mar Caribe y Pacífico sur, incluyendo los estados de Veracruz, Tabasco, Campeche, Yucatán, Quintana Roo, Oaxaca y Chiapas (Reyna et al. 2014). En estos últimos dos estados, los bosques mesófilos de montaña por debajo de los 2 mil metros de altitud también albergaron poblaciones de pecaríes de labios blancos hasta hace algunas décadas (March 1993; Naranjo 2002; Reid 1997; Sowls 1997). 
El pecarí de labios blancos se distingue considerablemente de otros mamíferos neotropicales por las grandes manadas que llega a formar y que pueden sobrepasar los 100 individuos (Sowls 1997). El tamaño promedio de los grupos registrados en México es de 13 - 28 individuos (Naranjo 2002; Reyna 2002). Su densidad poblacional se ha estimado entre 1 y 15 individuos $/ \mathrm{km}^{2}$, aunque con frecuencia estas cifras se encuentran por debajo de 5 ind $/ \mathrm{km}^{2}$ en sitios con cacería persistente (Bodmer et al. 1997; Naranjo et al. 2004a; Sowls 1997). Este mamífero requiere para sobrevivir de áreas forestales extensas (>10,000 ha) y sin actividad humana, preferentemente selvas altas y medianas húmedas, así como bosques bajos inundables (March 2005; Mayer y Wetzel 1987; Reyna 2007).

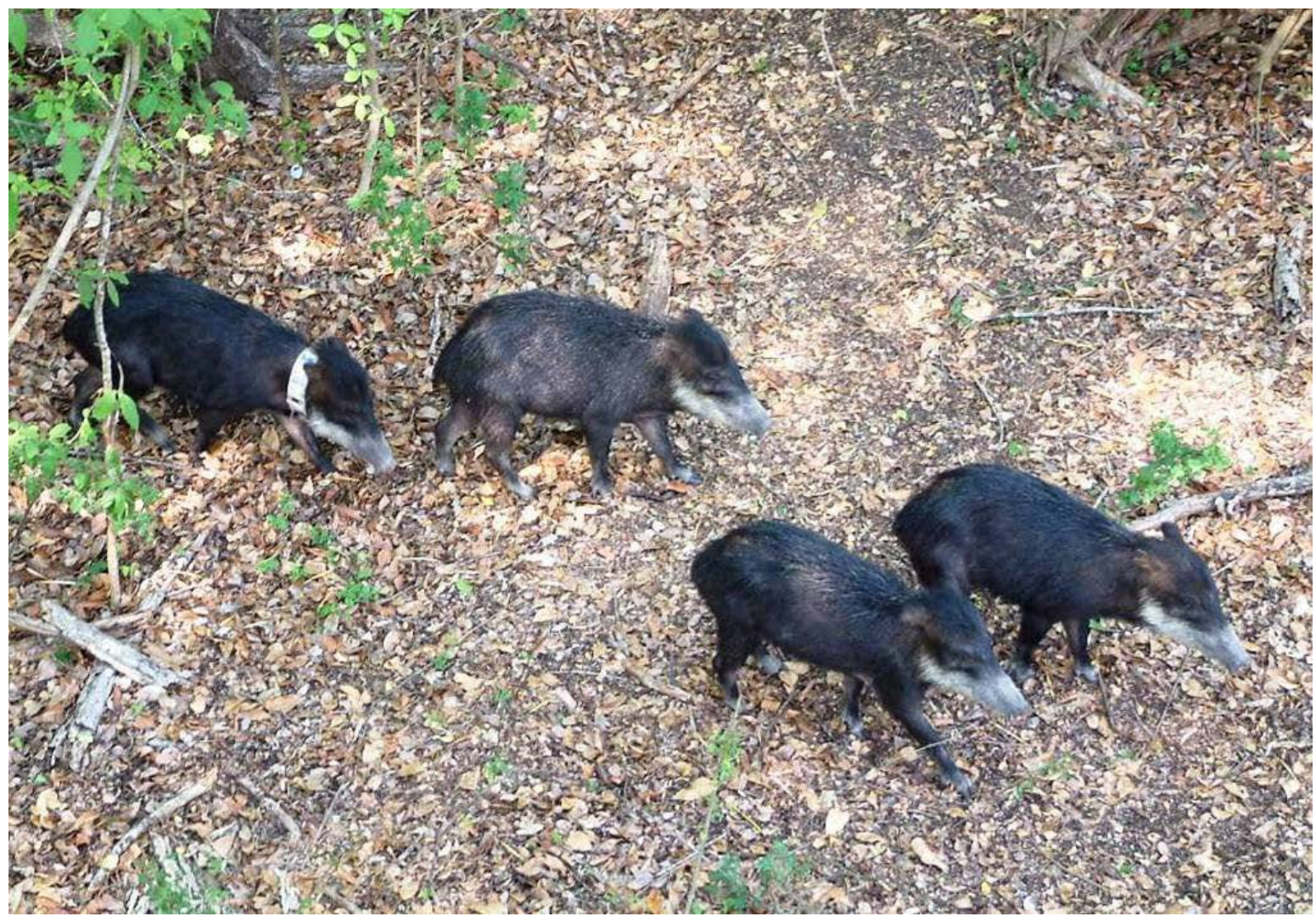

Figura 2. Pecaríes de labios blancos (Tayassu pecari). Reserva de la Biosfera Calakmul, Campeche. Foto: Rafael Reyna.

En la actualidad esta especie prácticamente ha desaparecido de Tabasco, y en Veracruz (Uxpanapa) y Yucatán (Dzilam) sobreviven pequeñas poblaciones aisladas (Naranjo et al. 2013). Solo los estados de Campeche, Chiapas, Oaxaca y Quintana Roo conservan poblaciones importantes en las Reservas de la Biosfera de Calakmul, Montes Azules, región de Los Chimalapas y Reserva de la Biosfera de Sian Ka'an, respectivamente (Lira y Briones 2011; Naranjo et al. 2013; Reyna et al. 2009). En Quintana Roo además sobreviven algunas poblaciones pequeñas en ejidos forestales cercanos a la Reserva de Sian Ka'an y al sur del estado y en Campeche en los alrededores de la Reserva de Calakmul (March 1990; Naranjo et al. 2013; Quijano 2001; Reyna et al. 2009). La superficie total estimada en la que subsiste la especie en México es de alrededor de 2 millones de hectáreas $\left(20,000 \mathrm{~km}^{2}\right)$, lo que apenas representa un poco más del $1 \%$ del territorio nacional (Naranjo y Reyna 2007). Existe una intensa cacería sin control de esta especie en casi toda su área de distribución, pues su carne es muy apreciada por los pobladores del medio rural en el sureste de México (Escamilla et al. 2000; Naranjo et al. 2004b; Weber 2000). Este hecho aunado a la severa deforestación ocurrida durante las últimas décadas en el sureste del país han ocasionado que el pecarí de labios blancos probablemente sea el ungulado tropical más raro de México en la actualidad (Naranjo y Bodmer 2007; Reyna et al. 2014). 
El objetivo central del presente estudio consistió en generar un diagnóstico sobre el estado actual de las poblaciones de tapir y pecarí de labios blancos en México, proponiendo una estrategia para su conservación. En particular, 1) se sistematizó información actualizada sobre la distribución, abundancia y estado de las poblaciones de ambas especies en el país; 2) se identificaron las amenazas más importantes para la supervivencia de las dos especies en México, elaborando una propuesta de acuerdo al Método de Evaluación de Riesgo (MER) para cada una de ellas; y 3) se propusieron líneas de acción útiles para integrar los Programas de Acción para la Conservación (PACE) de ambos mamíferos en México.

\section{Métodos}

\section{Área de estudio}

Las únicas poblaciones de tapir que han sido parcialmente estudiadas en México son las que se encuentran en la Selva Lacandona (Reserva Montes Azules), Sierra Madre de Chiapas (Reservas El Triunfo y La Sepultura), Los Chimalapas y Calakmul. En el caso del pecarí de labios blancos, el conocimiento de las poblaciones se limita a las Reservas Montes Azules y Calakmul, y a parte de Los Chimalapas. Por lo anterior, las áreas de estudio consideradas en esta investigación fueron aquellas para las cuales existía información publicada escasa o nula, y que además cuentan con gran extensión y buenas cualidades para soportar poblaciones importantes de al menos una de las dos especies objeto del estudio. Dado que algunos de los sitios de estudio propuestos son muy extensos, se seleccionaron localidades puntuales en cada uno de ellos para realizar las evaluaciones de campo (Figura 3, Tabla 1). Estas localidades se escogieron con base en su ubicación dentro de los polígonos propuestos, la existencia de fragmentos forestales extensos en sus territorios, la presencia confirmada o probable de alguna de las dos especies focales, su accesibilidad durante el período de estudio, y la anuencia de sus pobladores para permitir la realización de los muestreos y entrevistas.

Selva Zoque: La Selva Zoque incluye las regiones de Uxpanapa en Veracruz, Los Chimalapas en Oaxaca y la Selva El Ocote (Reserva de la Biosfera, $481 \mathrm{~km}^{2}$ ) en el estado de Chiapas, constituyendo una enorme ecoregión de más de $6,000 \mathrm{~km}^{2}$ caracterizada por su gran heterogeneidad topográfica (40 - 1,400 msnm) que origina variantes climáticas de tipo cálido húmedo y subhúmedo con abundantes lluvias en verano (Am, Aw y A(C)w; Arriaga et al. 2000; SEMARNAT 2001). La precipitación anual y la temperatura media anual varían entre 1,450 y $3,500 \mathrm{~mm}$, y 22 a $27^{\circ} \mathrm{C}$, respectivamente. Los principales tipos de vegetación son las selvas altas perennifolias, medianas subperennifolias, bajas caducifolias, bosques mesófilos de montaña, acahuales y pastizales inducidos. En la región se han registrado 149 especies de mamíferos incluyendo al tapir y al pecarí de labios blancos (Lira et al. 2012; SEMARNAT 2001), aunque en la Selva El Ocote no existen registros recientes de ésta última. Entre los principales problemas ambientales de la región están la deforestación, la expansión de la ganadería y los desmontes para el cultivo de estupefacientes, conflictos territoriales entre comunidades de Chiapas y Oaxaca, la apertura de carreteras, la cacería sin control y en particular los incendios forestales y quemas de acahuales y pastizales (Arriaga et al. 2000; Lira y Briones 2011; SEMARNAT 2001). Los muestreos en la Selva Zoque se llevaron a cabo en: 1) el Ejido La Fortaleza, municipio de Santa María Chimalapa, Oaxaca; 2) Ejido Loma de Oro y Predio El Jaguar cercano al poblado El Quince, municipio de Uxpanapa, Veracruz; y 3) cercanías del Ejido Emilio Rabasa en la Reserva de la Biosfera Selva El Ocote, municipio de Ocozocoautla, Chiapas.

La Fraylescana: Esta región enclavada en la Sierra Madre de Chiapas constituye un área protegida (Zona de Protección Forestal; $1,514 \mathrm{~km}^{2}$ ) y presenta relieve abrupto, con altitudes entre 250 y 2,550 msnm (Vargas y Escobar 2000). Los climas presentes incluyen el cálido y semicálido húmedo y subhúmedo (Am, Aw, A(C)w y A(C)m), y templado húmedo. La precipitación anual fluctúa entre 1,200 y 3,500 mm, y la temperatura media anual varía entre 18 y $24^{\circ} \mathrm{C}$. Los tipos de vegetación presentes 
son las selvas bajas caducifolias, selvas medianas subperennifolias, bosques de pino, bosques de encino y bosques mesófilos de montaña. En el área existen registros recientes de tapir, pero no de pecarí de labios blancos. Parte del área protegida está ocupada por pastizales inducidos, cafetales y cultivos de temporal. En el área existen procesos de deforestación por extracción de madera, expansión de la ganadería y la agricultura, cacería furtiva, erosión e incendios forestales (Pérez-Farrera et al. 2006). Los muestreos se efectuaron en la Finca Arroyo Negro, municipio de La Concordia, Chiapas.

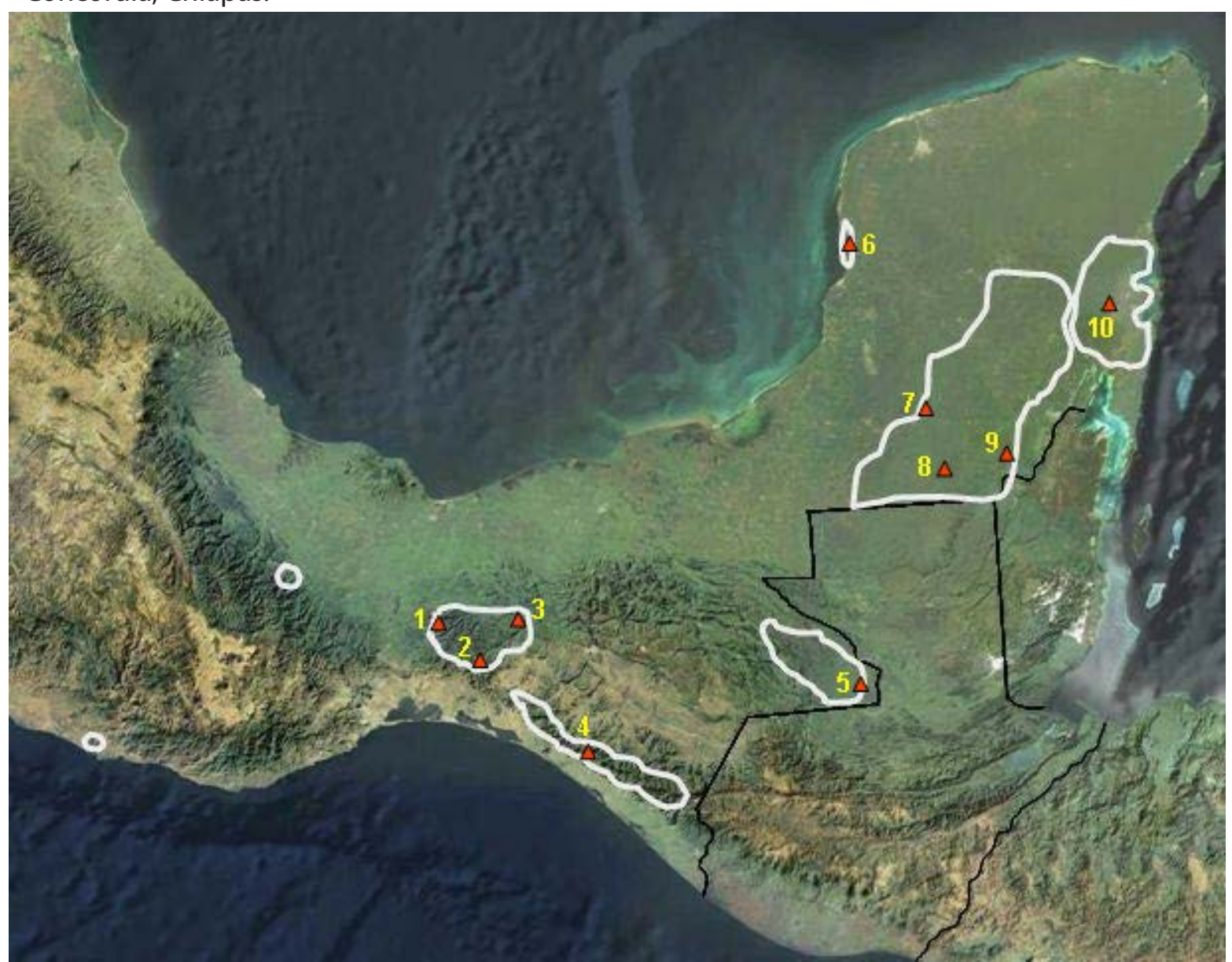

Figura 3. Ubicación de los sitios de estudio donde se evaluaron poblaciones de tapir y pecarí de labios blancos: 1) Uxpanapa, 2 Los Chimalapas, 3) Selva El Ocote, 4) La Fraylescana, 5) Marqués de Comillas, 6) Los Petenes, 7) Balam-kin y Balamkú, 8) Calakmul, 9) Ejidos forestales de Quintana Roo, 10) Sian Ka'an. Las áreas delineadas indican la distribución aproximada del tapir.

Marqués de Comillas: La subregión Marqués de Comillas de la Selva Lacandona está repartida en numerosos ejidos con habitantes provenientes de varias regiones de Chiapas y de otros estados del país. Su relieve es relativamente plano con algunos lomeríos hacia el suroeste y su altitud es de 150 a 200 msnm. El clima predominante es cálido húmedo (Am). La precipitación media anual es de casi 3,000 mm, y la temperatura media anual es de $25^{\circ} \mathrm{C}$. La vegetación predominante es selva alta perennifolia en diversos estadíos sucesionales, pastizales inducidos, cultivos de temporal y plantaciones de cacao. La fauna de la región incluye 118 mamíferos entre los que se encuentran el tapir y el pecarí de labios blancos (Naranjo 2002). Entre los factores de impacto ambiental en la región se encuentran el avance de la ganadería extensiva, la extracción maderera, la cacería sin control, los incendios forestales y las quemas de áreas agropecuarias (INE 2000b). El trabajo de campo se realizó en las cercanías del río Lacantún dentro de la Reserva de la Biosfera Montes Azules, municipio de Ocosingo, y en los Ejidos Adolfo López Mateos, Belisario Domínguez, Chajul, El Pirú, Flor del Marqués, Galacia, José López Portillo, Loma Bonita, Quiringuicharo, Playón de la Gloria, Reforma Agraria y Zamora Pico de Oro, municipio de Marqués de Comillas, Chiapas. 
Los Petenes: Esta área protegida abarca $3,824 \mathrm{~km}^{2}$ de ecosistemas terrestres y acuáticos en la planicie costera del norte de Campeche sujeta a inundaciones semipermanentes y estacionales. La topografía es plana y la altitud promedio es inferior a $10 \mathrm{msnm}$. En el área existen islas circulares de vegetación arbórea conocidas como petenes. Los tipos de clima predominantes son el cálido subhúmedo con lluvias en verano (Aw), y el semiseco cálido (BS'h'w). La temperatura y precipitación medias varían de 26 a $28^{\circ} \mathrm{C}$, y de 700 a 1,100 mm, respectivamente. La vegetación terrestre consiste en bosques de manglar, tulares, selva baja subperennifolia (inundable) y petenes. Se han observado 47 especies de mamíferos, con al menos un registro verificado de tapir (INE 2006; Naranjo et al. 2013). Las actividades con impacto en los ecosistemas de la reserva son la tala de manglares y selvas, la cacería furtiva y la pesca no regulada (INE 2006). El trabajo de campo se llevó a cabo en el interior de la Reserva de la Biosfera Los Petenes y el Ejido Zodzil, municipio de Hecelchakán, Campeche.

Calakmul, Balam-kiny Balamkú: Las Reservas Calakmul, Balam-kin y Balamkú abarcan una extensión conjunta de $7,760 \mathrm{~km}^{2}$. El relieve es predominantemente plano con algunos lomeríos de hasta 300 msnm. El manto de caliche que cubre la mayor parte de las tierras altas es poroso y absorbe gran

Tabla 1. Localización de los sitios de estudio donde se realizaron las evaluaciones poblacionales de tapir y pecarí de labios blancos.

\begin{tabular}{|c|c|c|c|c|}
\hline Estado & Sitio & Vértices aproximados & Tapir & Pecari \\
\hline Veracruz & 1. Región Uxpanapa & $\begin{array}{l}17^{\circ} 12^{\prime} \mathrm{N}-93^{\circ} 52^{\prime} \mathrm{O} \\
17^{\circ} 13^{\prime} \mathrm{N}-94^{\circ} 03^{\prime} \mathrm{O} \\
17^{\circ} 18^{\prime} \mathrm{N}-93^{\circ} 59^{\prime} \mathrm{O}\end{array}$ & $\mathrm{x}$ & $\mathrm{x}$ \\
\hline Oaxaca & 2. Región Los Chimalapas & $\begin{array}{l}16^{\circ} 54^{\prime} \mathrm{N}-94^{\circ} 13^{\prime} \mathrm{O} \\
16^{\circ} 54^{\prime} \mathrm{N}-94^{\circ} 30^{\prime} \mathrm{O} \\
17^{\circ} 00^{\prime} \mathrm{N}-94^{\circ} 02^{\prime} \mathrm{O} \\
17^{\circ} 06^{\prime} \mathrm{N}-94^{\circ} 30^{\prime} \mathrm{O}\end{array}$ & $\mathrm{x}$ & $\mathrm{x}$ \\
\hline Chiapas & 3. Reserva de la Biosfera Selva El Ocote & $\begin{array}{l}16^{\circ} 54^{\prime} \mathrm{N}-93^{\circ} 34^{\prime} \mathrm{O} \\
16^{\circ} 59^{\prime} \mathrm{N}-93^{\circ} 32^{\prime} \mathrm{O} \\
17^{\circ} 00^{\prime} \mathrm{N}-93^{\circ} 44^{\prime} \mathrm{O} \\
17^{\circ} 04^{\prime} \mathrm{N}-93^{\circ} 37^{\prime} \mathrm{O}\end{array}$ & $\mathrm{x}$ & \\
\hline Chiapas & 4. Zona de Protección Forestal La Fraylescana & $\begin{array}{l}15^{\circ} 51^{\prime} \mathrm{N}-93^{\circ} 15^{\prime} \mathrm{O} \\
15^{\circ} 52^{\prime} \mathrm{N}-93^{\circ} 09^{\prime} \mathrm{O} \\
15^{\circ} 54^{\prime} \mathrm{N}-93^{\circ} 15^{\prime} \mathrm{O} \\
15^{\circ} 56^{\prime} \mathrm{N}-93^{\circ} 09^{\prime} \mathrm{O}\end{array}$ & $x$ & \\
\hline Chiapas & 5. Selva Lacandona, Subregión Marqués de Comillas & $\begin{array}{l}16^{\circ} 06^{\prime} \mathrm{N}-90^{\circ} 48^{\prime} \mathrm{O} \\
16^{\circ} 06^{\prime} \mathrm{N}-90^{\circ} 51^{\prime} \mathrm{O} \\
16^{\circ} 15^{\prime} \mathrm{N}-90^{\circ} 47^{\prime} \mathrm{O} \\
16^{\circ} 17^{\prime} \mathrm{N}-90^{\circ} 50^{\prime} \mathrm{O}\end{array}$ & $\mathrm{x}$ & $x$ \\
\hline Campeche & 6. Reserva de la Biosfera Los Petenes & $\begin{array}{l}20^{\circ} 00 \mathrm{~N}-90^{\circ} 22^{\prime} \mathrm{O} \\
20^{\circ} 00 \mathrm{~N}-90^{\circ} 26^{\prime} \mathrm{O} \\
20^{\circ} 13 \mathrm{~N}-90^{\circ} 22^{\prime} \mathrm{O} \\
20^{\circ} 13 \mathrm{~N}-90^{\circ} 26^{\prime} \mathrm{O}\end{array}$ & $x$ & \\
\hline Campeche & 7. Reservas Estatales Balam-kin y Balamkú & $\begin{array}{l}18^{\circ} 39^{\prime} \mathrm{N}-90^{\circ} 31^{\prime} \mathrm{O} \\
18^{\circ} 39^{\prime} \mathrm{N}-90^{\circ} 36^{\prime} \mathrm{O} \\
18^{\circ} 45^{\prime} \mathrm{N}-90^{\circ} 31^{\prime} \mathrm{O} \\
18^{\circ} 45^{\prime} \mathrm{N}-90^{\circ} 36^{\prime} \mathrm{O}\end{array}$ & $\mathrm{x}$ & $\mathrm{x}$ \\
\hline Campeche & 8. Reserva de la Biosfera Calakmul & $\begin{array}{l}18^{\circ} 06^{\prime} \mathrm{N}-89^{\circ} 56^{\prime} \mathrm{O} \\
18^{\circ} 06^{\prime} \mathrm{N}-90^{\circ} 19^{\prime} \mathrm{O} \\
18^{\circ} 26^{\prime} \mathrm{N}-89^{\circ} 56^{\prime} \mathrm{O} \\
18^{\circ} 26^{\prime} \mathrm{N}-90^{\circ} 17^{\prime} \mathrm{O}\end{array}$ & $\mathrm{x}$ & $x$ \\
\hline Quintana Roo & 9. Ejidos forestales del centro y sur & $\begin{array}{l}18^{\circ} 16^{\prime} \mathrm{N}-88^{\circ} 54^{\prime} \mathrm{O} \\
18^{\circ} 16^{\prime} \mathrm{N}-88^{\circ} 57^{\prime} \mathrm{O} \\
18^{\circ} 19^{\prime} \mathrm{N}-88^{\circ} 54^{\prime} \mathrm{O} \\
18^{\circ} 19^{\prime} \mathrm{N}-88^{\circ} 57^{\prime} \mathrm{O}\end{array}$ & $\mathrm{x}$ & $x$ \\
\hline Quintana Roo & 10. Reserva de la Biosfera Sian Ka'an & $\begin{array}{l}19^{\circ} 08^{\prime} \mathrm{N}-87^{\circ} 57^{\prime} \mathrm{O} \\
19^{\circ} 08^{\prime} \mathrm{N}-88^{\circ} 01^{\prime} \mathrm{O} \\
19^{\circ} 11^{\prime} \mathrm{N}-87^{\circ} 57^{\prime} \mathrm{O} \\
19^{\circ} 11^{\prime} \mathrm{N}-88^{\circ} 01^{\prime} \mathrm{O}\end{array}$ & $\mathrm{x}$ & $x$ \\
\hline
\end{tabular}


parte del agua de lluvia, por lo que no existen corrientes de agua permanentes y ésta se encuentra disponible solamente en lagunas dispersas de baja profundidad llamadas "aguadas". El clima es cálido subhúmedo (Aw), la precipitación varía de 600 a $1200 \mathrm{~mm}$ y la temperatura media anual es de 24 a $26^{\circ} \mathrm{C}$. Las selvas altas y medianas perennifolias y subperennifolias dominan el paisaje, aunque también están presentes selvas bajas caducifolias inundables y vegetación acuática. Entre las casi 100 especies de mamíferos presentes se encuentran el tapir y el pecarí de labios blancos (INE 2000a; Reyna 2007). La escasez de agua en la región limita la expansión de la agricultura y la ganadería, pero persisten la extracción forestal, la cacería ilegal, los incendios forestales y la apertura de carreteras (INE 2000a). Los muestreos de abundancia se hicieron en las cercanías de la zona arquelógica de Calakmul y en el interior de la Reserva de Balam-kin, ambos en el municipio de Calakmul, Campeche. Las entrevistas se realizaron en los Ejidos Nuevo Becal, 20 de Noviembre y Conhuas, todos del mismo municipio.

Sian Ka'an y Ejidos Forestales de Quintana Roo: La gran extensión forestal de esta región (> $6,000 \mathrm{~km}^{2}$ ) la hacen una de las más importantes del país desde el punto de vista de conservación del tapir y el pecarí de labios blancos. El relieve es predominantemente plano, los suelos son pedregosos y someros, y el clima es cálido subhúmedo con lluvias en verano (Aw). La temperatura y precipitación medias son de $26.5^{\circ} \mathrm{C}$ y $1,200 \mathrm{~mm}$, respectivamente. Dominan el paisaje las selvas medianas subperennifolias, selvas bajas subcaducifolias y caducifolias, bosques de manglar, acahuales y dunas costeras. Se han registrado en la región 114 especies de mamíferos, entre ellos el tapir y el pecarí de labios blancos (Escobedo 2011; SEDESOL 1993). Entre los problemas ambientales de esta región se encuentran la extracción forestal sin control, los incendios, la construcción de autopistas y los desarrollos turísticos a gran escala (Lozano y Olivares 2011). El trabajo de campo se llevó a cabo en los ejidos Chanká Veracruz y X-Hazil (municipio de Felipe Carrillo Puerto) y Caobas (municipio de Othón P. Blanco), Quintana Roo.

Trabajo de campo. El trabajo de campo de este estudio se desarrolló de noviembre 2010 a noviembre 2012. Durante los primeros meses se hizo una recopilación y sistematización de la información publicada (artículos y libros) y no publicada (tesis, informes de investigación, bases de datos) sobre las poblaciones mexicanas de las dos especies bajo estudio. Para ello se recurrió a los sistemas de información documental de instituciones como El Colegio de la Frontera Sur (ECOSUR), la Universidad Nacional Autónoma de México (UNAM), la Universidad de Ciencias y Artes de Chiapas (UNICACH), los Grupos de Especialistas en Tapires y Pecaríes de la Unión Internacional para la Conservación de la Naturaleza (UICN), y el Sistema Nacional de Información sobre Biodiversidad (SNIB) de la Comisión Nacional para el Conocimiento y Uso de la Biodiversidad (CONABIO).

\section{Evaluaciones poblacionales}

En cada sitio de trabajo se realizó una evaluación rápida de la presencia y abundancia relativa de tapir y pecarí de labios blancos mediante tres técnicas complementarias con ayuda de pobladores locales, técnicos y tesistas. La primera técnica consistió en la colocación de una retícula de $3 \times 3$ cámaras-trampa (modelos Moultrie D55 y D55IR) ubicadas a $1 \mathrm{~km}$ de distancia entre sí por cada localidad. Las cámaras se ajustaron a una altura de entre 50 y $70 \mathrm{~cm}$ del suelo en troncos de árboles, y se programaron para tomar series de 3 fotografías con tiempo de espera de 1 minuto entre eventos. Cada retícula de cámaras permaneció entre 25 y 40 días en un mismo sitio de muestreo. A partir de las imágenes capturadas se calcularon índices de abundancia (tasas fotográficas: número de fotos/ número de cámaras/ número de días de muestreo x 100; Conroy 1996; Cutler y Swann 1999; O'Connell et al. 2011) para cada especie en cada sitio de trabajo.

Durante el tiempo que las cámaras-trampa permanecieron funcionando en el campo se aplicó una segunda técnica de muestreo, consistente en recorridos diurnos a lo largo de dos a tres 
transectos lineales de 2-5 km de longitud por localidad. Durante estos recorridos se hicieron conteos de individuos o rastros (huellas y excretas frescas) de cualquiera de las dos especies. Cada transecto fue recorrido cuando menos dos veces durante el estudio, anotando el número de individuos y rastros observados de cada especie, así como la fecha, posición geográfica (coordenadas) y las características del hábitat (tipo de vegetación, geomorfología, presencia de cuerpos de agua y distancia a asentamientos humanos) correspondientes a cada registro. A partir de las observaciones logradas en los transectos se estimaron índices de abundancia relativa (número de rastros o individuos/ $10 \mathrm{~km}$ recorridos; Conroy 1996; Naranjo 2000).

La tercera y última técnica de muestreo fue la aplicación de entrevistas semi-estructuradas a pobladores de las comunidades rurales más cercanas a las áreas visitadas. Se utilizó un cuestionario guía para recopilar información sobre la presencia y abundancia estimada de tapires y pecaríes de labios blancos, en el que se incluyeron preguntas sobre la antigüedad de los avistamientos de ambas especies en la localidad, así como las características del hábitat (arriba citadas) en el que fueron observados los ejemplares, la existencia de cacería y otras amenazas potenciales para las especies objeto del estudio. Para evitar dudas en la identificación de las especies por los entrevistados, se mostraron ilustraciones a color de las mismas tomadas de guías de campo (Emmons y Feer 1997; Reid 1997). Cuando se obtuvieron referencias de animales cazados recientemente en una comunidad, se procuró localizar y examinar los restos de los mismos (cráneos o pieles) para corroborar la especie y estimar la categoría de edad (juvenil o adulto) con base en las dimensiones de las partes disponibles (Naranjo y Bodmer 2002). Los restos localizados fueron depositados en la colección mastozoológica de El Colegio de la Frontera Sur en San Cristóbal de Las Casas, Chiapas.

\section{Análisis de la información}

La distribución espacial del tapir y el pecarí de labios blancos se determinó en base a su presencia o ausencia en cada transecto y sitio de estudio. Se registró la presencia actual de las dos especies en cada sitio a través de las cámaras-trampa, los recorridos para observación de animales o sus rastros, y las entrevistas a pobladores locales. Estas últimas aportaron también información sobre la distribución histórica de ambas especies. Una vez confirmada o descartada la presencia real de tapires y pecaríes de labios blancos en cada sitio de estudio, con la ayuda de un sistema de información geográfica (ArcView; ESRI 2008) se elaboraron mapas de distribución actual verificada de cada especie. Los factores de amenaza a las especies bajo estudio (deforestación, fragmentación, incendios, cacería y presencia de animales domésticos) se identificaron en cada sitio de estudio y se clasificaron utilizando una escala semi-cuantitativa (bajo = 0-1 factores presentes; medio = 2-3; alto $>3$ ).

Método de Evaluación de Riesgo (MER). Como resultado de la recopilación de información y las evaluaciones de campo arriba descritas, se generó un diagnóstico del estado de las poblaciones de tapir y pecarí de labios blancos en el país que se plasmó en un protocolo denominado "Método de Evaluación de Riesgo" (MER; SEMARNAT 2010). Para realizar estos protocolos se revisó la literatura disponible sobre ambas especies en todas sus áreas de distribución. La amplitud de la distribución de cada especie en el país (criterio A del MER) se estimó con base en los mapas de distribución real generados en este proyecto. El estado del hábitat (criterio B) se evaluó a partir tanto de la información sobre factores de amenaza y características del hábitat obtenidas en este estudio, como del análisis de la literatura disponible sobre los requerimientos vitales de ambos mamíferos y el estado de los ecosistemas en cada sitio de trabajo. La vulnerabilidad biológica intrínseca (criterio C) de cada especie se derivó de la literatura: 2 crías/hembra/año para el pecarí de collar (vulnerabilidad media; Bodmer et al. 1997; March 2005), y 0.5 crías/hembra/año para el tapir (vulnerabilidad alta; March y Naranjo 2005; Naranjo 2009). El impacto de la actividad humana sobre las dos especies (criterio D) se determinó con base en el análisis de factores de amenaza generado en el presente proyecto, 
así como en la revisión de literatura disponible y en las experiencias de más de dos décadas de investigación realizada por los miembros del equipo de trabajo en el sureste del país.

\section{Resultados y Discusión}

Distribución. Puesto que ya existían modelos recientes de la distribución potencial de ambas especies en el país (realizados mediante algoritmos GARP y otros; Ceballos 2008: Mendoza et al. 2013; Nolasco 2009), y considerando que dichos modelos son susceptibles a la sobreestimación de la distribución real (Loiselle et al. 2003), el análisis de distribución en este estudio se concentró en la verificación selectiva en campo de la presencia actual de los dos ungulados. Durante la investigación se lograron 105 registros de tapir $(n=79)$ y pecarí de labios blancos $(n=26$; Tabla 2). Los muestreos de distribución realizados durante el proyecto permitieron obtener evidencias de la presencia de tapires en los 10 sitios seleccionados (Figura 4), lo que coincide plenamente con el modelo de distribución del hábitat potencial de esta especie en México desarrollado por Mendoza et al. (2013). En contraste, el pecarí de labios blancos solamente se detectó en seis de ellos, estando ausente de las regiones de la Selva El Ocote, La Fraylescana (Sierra Madre de Chiapas) y Los Petenes, Campeche (Figura 5 y Tabla 2). Aunque no se logró obtener evidencia de la presencia del pecarí de labios blancos en Los Chimalapas durante este proyecto, existe información previa de su presencia y abundancia en la región (Lira y Briones 2011; Lira et al. 2012). Lo anterior concuerda con propuestas previas sobre la disminución del hábitat para este pecarí (March 2005; Reyna et al. 2014), y fortalece la percepción de los pobladores de la mayoría de las localidades de estudio que se describe a continuación.

El total acumulado de entrevistas realizadas con pobladores de 20 comunidades rurales en los sitios de estudio fue de 111. Los análisis de la información relacionada con la distribución de ambas especies recabada en las entrevistas confirmaron que el tapir está presente en todos los sitios de estudio y en 18 de las 20 comunidades visitadas, faltando solamente en Benemérito de la Américas y Quiringuicharo (Selva Lacandona) desde hace 10 - 20 años. Por el contrario, el pecarí

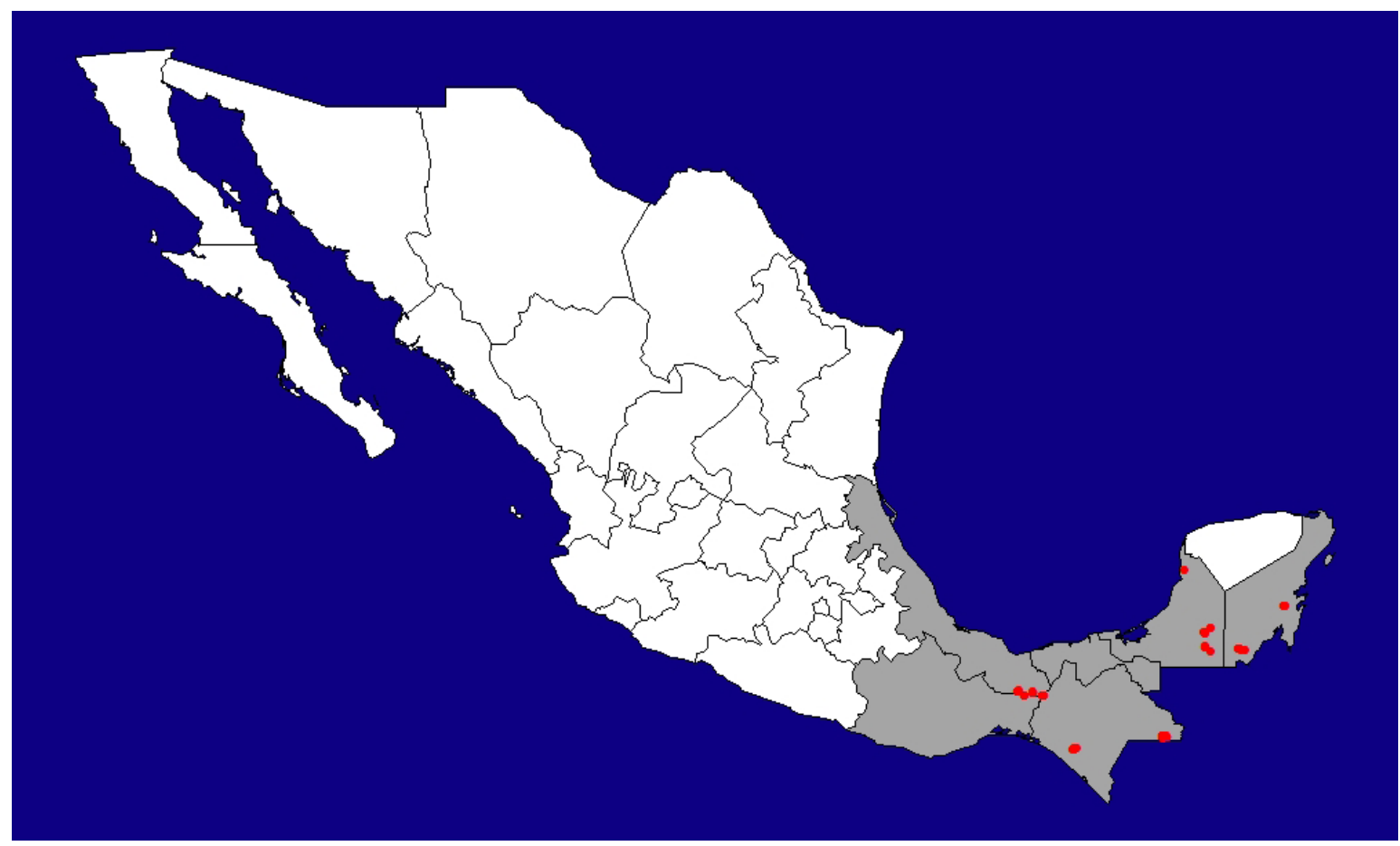

Figura 4. Distribución actual del tapir (Tapirus bairdii) en México con base exclusivamente en los registros obtenidos $(n=79)$ en el trabajo de campo del presente proyecto. Mapa: Fredy Falconi Briones. 
de labios blancos solamente persiste en siete de los 10 sitios de estudio (ausente de La Fraylescana, Selva El Ocote y Los Petenes) y en 12 de las 20 comunidades visitadas. Prácticamente todos (100\%) los pobladores consultados conocen a las dos especies ya sea por observación directa de animales vivos o cazados, o por referencia directa de sus padres y abuelos. La gran mayoría (81\%; 90/111) de los entrevistados aseguró que el tapir está presente en sus comunidades, mientras que el $15.3 \%$ (17/111) afirmó que esta especie ya no está presente, y el restante $3.7 \%(4 / 111)$ dijo no estar seguro. En el caso del pecarí de labios blancos, solamente el $34.2 \%$ (38/111) de los entrevistados dijo que la especie aún está presente en su comunidad, mientras que la mayoría de ellos $(59.5 \%$; 66/111) afirmó que ya no está presente, y el restante 6.3 \% (7/111) no sabía. La percepción de los habitantes de los sitios de estudio evaluada a través de las entrevistas realizadas sugiere que el pecarí de labios blancos tiene actualmente una distribución notablemente más restringida que la del tapir en el país, lo cual coincide plenamente con los registros fotográficos y avistamientos de ejemplares y sus rastros logrados en este estudio.

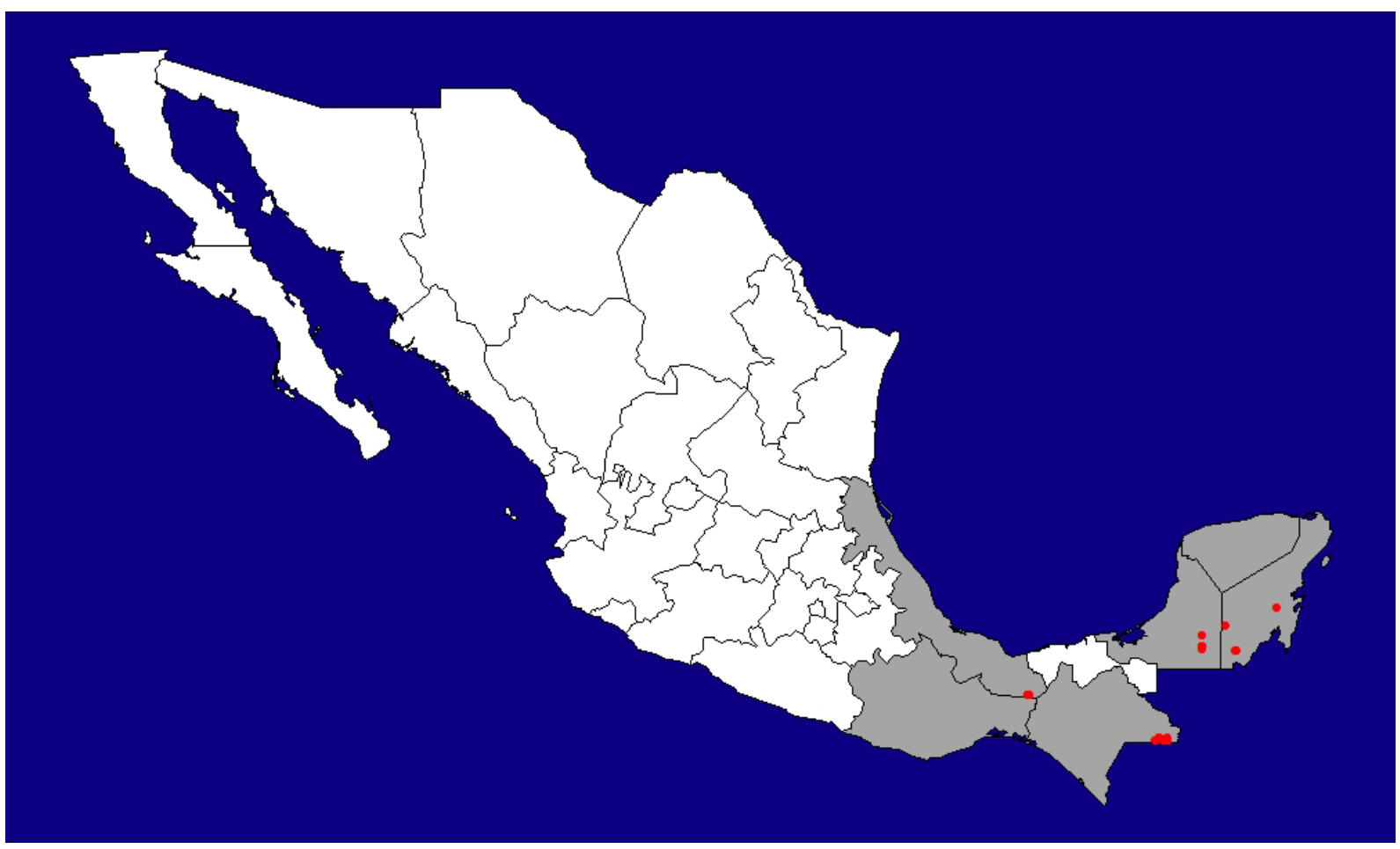

Figura 5. Distribución actual del pecarí de labios blancos (Tayassu pecari) en México con base exclusivamente en los registros obtenidos $(n=26)$ en el trabajo de campo del presente proyecto. Mapa: Fredy Falconi Briones.

Los registros de presencia del tapir (Figura 6) logrados en el presente trabajo confirman que sus áreas de distribución actuales y verificadas en México se encuentran en los estados de Campeche (Reservas de Calakmul, Balamkú, Balam-kin, Los Petenes y Laguna de Términos); Chiapas (Reservas Selva El Ocote, El Triunfo, La Sepultura, Lacantún y Montes Azules, Comunidad Lacandona y algunos ejidos de Marqués de Comillas y de la Sierra Madre); Oaxaca (Los Chimalapas, Bajo Río Verde, Sierra Veinte Cerros y Sierra de Ixtlán de Juárez); Quintana Roo (Reserva Sian Ka'an y ejidos forestales del centro y sur), y Veracruz (cuenca alta del río Uxpanapa). En el caso del pecarí de labios blancos, las áreas de distribución verificadas coinciden con las del tapir, a excepción de la Reservas Los Petenes y Selva El Ocote, y la Sierra Madre de Chiapas. Entre éstas áreas, por su gran extensión y buena calidad de hábitat para las dos especies destacan las de Calakmul-Balamkú-Balam-kin (Figura 7), Montes Azules-Lacantún, Los Chimalapas, y Sian Ka'an-ejidos forestales del centro de Quintana Roo, las cuales pueden considerarse como críticas para la conservación de ambas especies en el país. 
Es muy importante señalar que, gracias a la comunicación con investigadores de otras instituciones, se ha confirmado recientemente la presencia del pecarí de labios blancos mediante registros con cámaras-trampa en las cercanías de la Laguna de Términos en el municipio de Palizada, y en los ejidos Reforma Agraria y Pixtún, municipio de Champotón, ambos en la porción occidental del estado de Campeche (Mircea Hidalgo, Universidad Juárez Autónoma de Tabasco, com. pers. 2012). Otro hallazgo muy relevante de esta especie con la misma técnica es el logrado en septiembre de 2011 en el Rancho El Edén, dentro de la Reserva de Dzilam de Bravo, en la costa central de Yucatán (Luis Pereira y Carlos Alcérreca, BIOCENOSIS, A.C., com. pers. 2012).

Estos nuevos registros hacen evidente la necesidad de realizar exploraciones más detalladas de los sitios señalados, así como de otras áreas (i. e. Sierra Madre de Chiapas, Sierra Mixe de Oaxaca, Uxpanapa, Laguna de Términos, Champotón, Los Petenes y áreas costeras de Yucatán), lo que permitirá verificar la presencia o ausencia de tapires y pecaríes de labios blancos y evaluar el estado de conservación de las poblaciones encontradas así como la posible conectividad de éstas con otras más grandes, de manera que se tengan mayores elementos para hacer un diagnóstico realista sobre su persistencia a largo plazo.

Abundancia. Los índices de abundancia estimados a partir de muestreos en transectos lineales variaron entre 0.26 - 4.52 rastros de tapires, y 0.15 - 0.63 rastros de pecaríes de labios blancos por $10 \mathrm{~km}$ recorridos (Figuras 7 y 8 , Tabla 3). En el caso del tapir, los valores de abundancia obtenidos en este estudio se encuentran dentro del rango de abundancias estimadas en estudios previos realizados en México (0.05 a 8.1 rastros por 10 km; Lira et al. 2004; Naranjo 2009; Naranjo y Bodmer 2002; Naranjo y Cruz 1998; Reyna y Tanner 2007; Tejeda et al. 2009) y en otros países

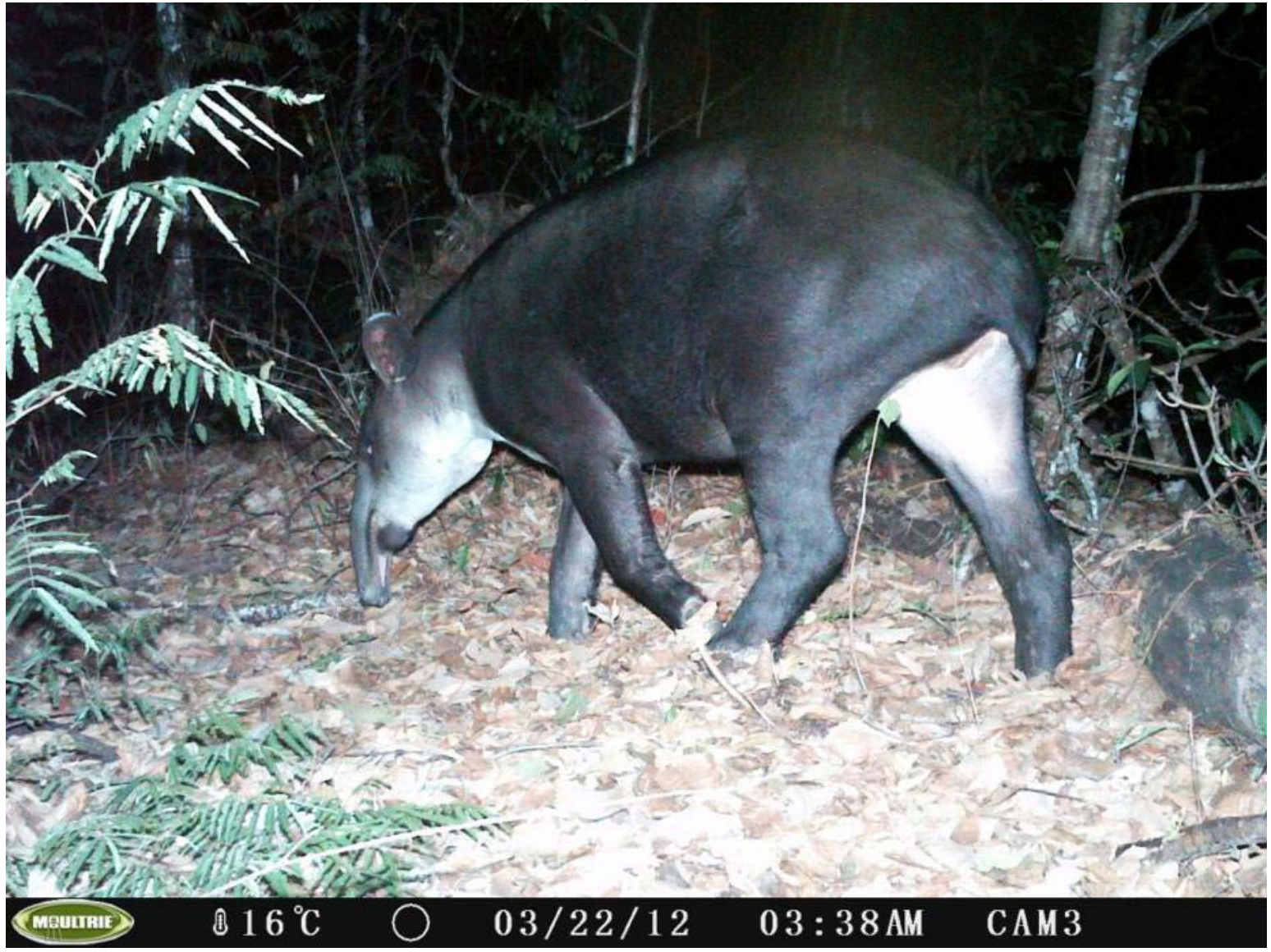

Figura 6. Hembra adulta de tapir (Tapirus bairdii) fotografiada durante el proyecto con cámara-trampa en la Reserva Balam-kin, Campeche. Foto: Eduardo Naranjo, Fredy Falconi y Saúl Amador. 
de Mesoamérica (1.5 a 6.6 rastros por 10 km; Flesher 1999; Naranjo 1995). En el caso del pecarí de labios blancos, las estimaciones realizadas en los sitios de estudio de la Selva Lacandona y Balamkin se encuentran ligeramente por debajo del rango de abundancia encontrado en estudios previos en el país (0.21 a 2.0 rastros por 10 km; Naranjo et al. 2004b; Reyna y Tanner 2007; Tejeda et al 2009), mientras que el resto se ubican dentro de dicho intervalo. Los índices de abundancia estimados mediante foto-trampeo se ubicaron entre 0.38 - 2.00 (tapir) y 0.17 - 1.33 (pecarí) fotografías por 100 días-cámara (Figuras 7 y 8; Tabla 3). Las abundancias de ambos mamíferos estimadas con esta técnica fueron similares comparadas con las registradas en estudios previos en Los Chimalapas ( 0.8 y 2.5 fotografías por 100 días-cámara para tapir y pecarí, respectivamente; Lira y Briones 2012) y en general más bajas que las estimadas en localidades de Centro y Sudamérica ( 1 a 20 fotografías por 100 días-cámara; Harmsen et al. 2010; Silveira et al. 2003). No obstante lo anterior, al considerar las variantes metodológicas y ambientales (i. e. distintos hábitat, diseños y esfuerzos de muestreo, diferentes modelos de cámaras) aplicadas en los diversos estudios realizados, resulta difícil formular conjeturas convincentes que expliquen las diferencias encontradas en las abundancias relativas de ambas especies entre dichos estudios y el presente.

Aunque los mayores números de registros totales de ambas especies se obtuvieron en la Selva Lacandona, ésta no presentó en todos los casos los mayores índices de abundancia una vez considerado el gran esfuerzo de muestreo invertido (kilómetros recorridos y cámaras-trampa/día). Las mayores abundancias relativas de tapir estimadas mediante rastros y avistamientos directos se registraron en La Fraylescana, Uxpanapa y Los Chimalapas, mientras que las tasas fotográficas más elevadas para esta especie se obtuvieron en Calakmul, Uxpanapa, la Selva Lacandona y Balam-kin. En el caso del pecarí de labios blancos, las mayores abundancias a partir de rastros y avistamientos directos se estimaron en Sian Ka'an, Calakmul y Uxpanapa, mientras que los mayores valores a partir de foto-trampeo se obtuvieron en Calakmul y Balam-kin. Es interesante señalar que en este estudio no se lograron obtener fotografías con las cámaras-trampa en la Selva El Ocote, Los Petenes y Sian Ka'an para ninguna de las dos especies, y además en La Fraylescana, los ejidos forestales de Quintana Roo, y Los Chimalapas para el pecarí de labios blancos. Se esperaría que los sitios donde se registraron las mayores abundancias coincidieran en poseer grandes extensiones de bosque tropical húmedo con buena disponibilidad de agua y recursos alimentarios, además de no presentar elevada presión de cacería por los pobladores locales. Sin embargo, las abundancias tan diversas estimadas en este trabajo sugieren que las condiciones del hábitat para los tapires y los pecaríes de labios blancos en México son muy heterogéneas debido a una variedad de factores ambientales (clima, geomorfolgía, vegetación y disponibilidad de agua, entre otros) y sociales (usos del suelo, presión de cacería y presencia de asentamientos humanos, entre otros), lo que coincide con las apreciaciones de

Tabla 2. Número de registros de tapir y pecarí de labios blancos (LB) y entrevistas aplicadas en los sitios de estudio visitados en el período mayo - octubre 2012 y cifras totales. La presencia de al menos una de las dos especies ha sido confirmada en todos los sitios.

\begin{tabular}{l|c|c|c|c}
\hline \multicolumn{1}{c}{} & $\begin{array}{c}\text { Registros } \\
\text { Sitio }\end{array}$ & Registros & Registros & Totales \\
\hline Selva Lacandona & 22 & 10 & 32 & 58 \\
Selva El Ocote & 1 & 0 & 1 & 5 \\
La Fraylescana & 7 & 0 & 7 & 4 \\
Uxpanapa & 12 & 5 & 17 & 7 \\
Los Chimalapas & 14 & 0 & 14 & 6 \\
Ejidos Forestales de Quintana Roo & 7 & 2 & 9 & 6 \\
Sian Ka'an & 4 & 1 & 5 & 10 \\
Calakmul & 6 & 7 & 13 & 6 \\
Balam-kin & 5 & 1 & 6 & 4 \\
Los Petenes & 1 & 0 & 1 & 5 \\
\hline
\end{tabular}




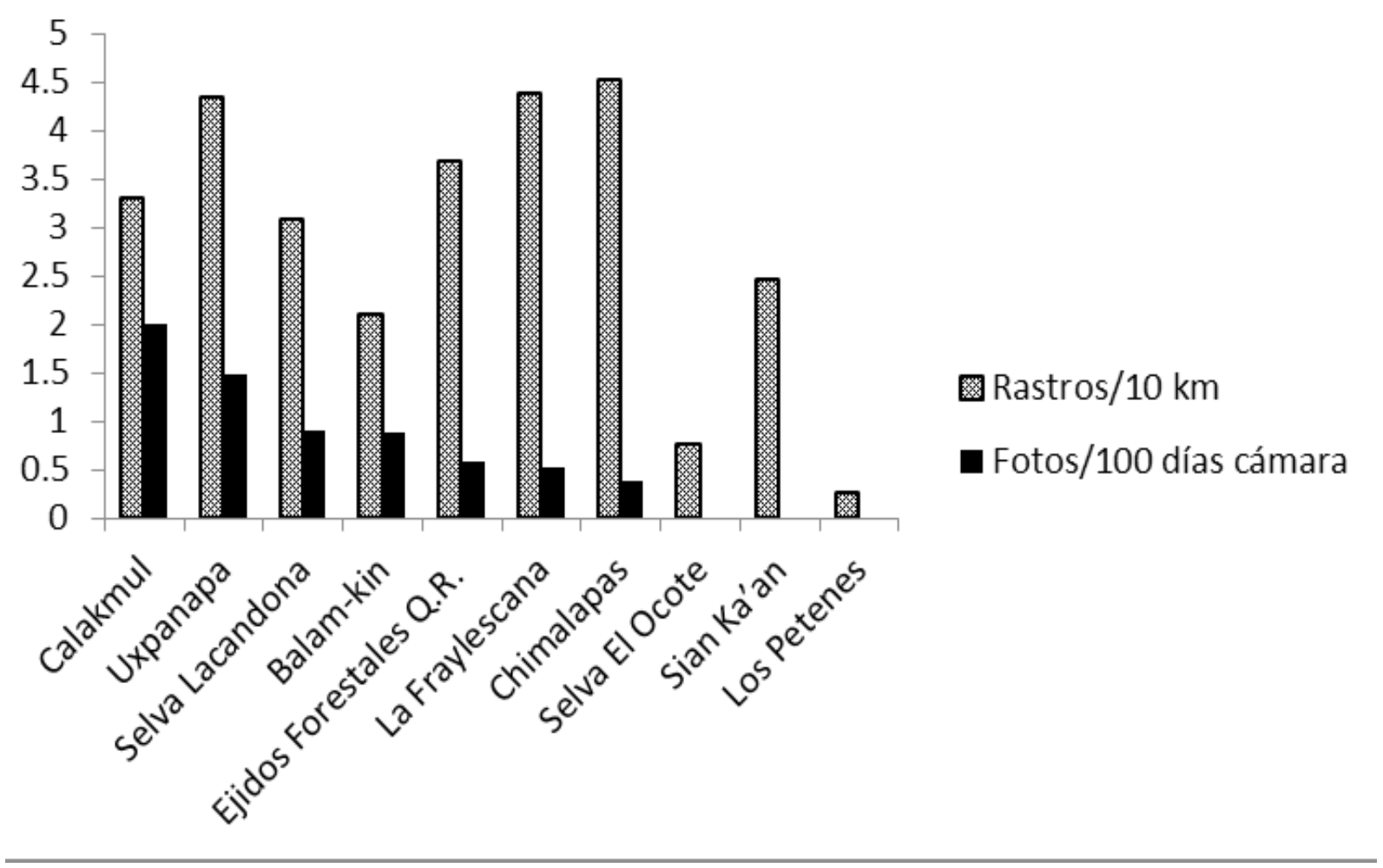

Figura 7. Abundancia del tapir (Tapirus bairdii) estimada mediante foto-trampeo y conteos de rastros en las localidades de estudio.

Naranjo $(2009,2014)$ y Reyna et al. (2014). Además, debe considerarse que los métodos de estudio empleados pueden ser afectados por las condiciones meteorológicas durante los períodos de muestreo (Naranjo 2000; O'Connell et al. 2011), de manera que los animales pueden modificar sus patrones de actividad, por ejemplo, si se presentan lluvias abundantes o días extremadamente calurosos, como sucedió en algunos de los sitios de estudio.

Amenazas. Las visitas al campo y las entrevistas con pobladores locales realizadas en este estudio permitieron atestiguar que entre las amenazas principales para la conservación del tapir y el pecarí de labios blancos en sus áreas de distribución actuales destacan en primer lugar la deforestación y fragmentación del hábitat (fundamentalmente selvas húmedas y subhúmedas), seguida de la cacería sin control (más notable para el pecarí) y otros factores como los incendios forestales, la sequía, las inundaciones, los huracanes y posiblemente las enfermedades transmitidas por animales domésticos (Naranjo et al. 2013; Reyna et al. 2014; Romero et al. 2008). Al respecto, la mayoría de los residentes entrevistados en los sitios de estudio opinaron que tanto el tapir (94\%) como el pecarí de labios blancos (78\%) se encuentran en situación de riesgo dentro de sus comunidades. Las amenazas más importantes para ambas especies percibidas por los entrevistados fueron la deforestación y fragmentación del hábitat, citadas por aproximadamente el $75 \%$ de los entrevistados, seguida por la cacería sin control (38\%), los incendios forestales (18\%) y otros factores como la sequía, las inundaciones, las carreteras y las enfermedades (6\%). Las proporciones de los primeros dos factores (pérdida de hábitat y cacería) coinciden plenamente con nuestras observaciones en los sitios de trabajo, donde en muchos casos fue evidente el avance de la frontera agropecuaria sobre áreas hasta hace poco cubiertas con selvas maduras, además de la detección de evidencias de cacería (por ejemplo, cartuchos vacíos, plataformas construidas en árboles para esperar a las presas y cráneos perforados, entre otras). Sin embargo, fue sorprendente la elevada proporción de entrevistados que perciben a los incendios como una amenaza importante para las dos especies 
objeto del estudio, particularmente en la Selva El Ocote, Uxpanapa y Los Chimalapas, donde en las pasadas dos décadas se han presentado varios eventos de gran magnitud (Naranjo 2012; Ortega del Valle et al. 2012). En la Tabla 4 se muestran en orden de importancia las principales amenazas observadas tanto por el equipo de trabajo del proyecto como por los pobladores entrevistados en las comunidades visitadas. Por la cantidad observada de factores de amenaza, las áreas de mayor riesgo para las poblaciones evaluadas de tapir son Los Petenes, Uxpanapa y Los Chimalapas, en tanto que para el pecarí de labios blancos son también las dos últimas y además los ejidos forestales de Quintana Roo y ejidos del Marqués de Comillas en la Selva Lacandona (Tabla 4).

A pesar de la variedad e intensidad de las amenazas observadas para el tapir y el pecarí de labios blancos en los sitios de estudio, se recopilaron también algunos signos alentadores de recuperación de poblaciones en sitios puntuales como el Ejido Playón de la Gloria (Selva Lacandona), donde desde el año 2010 se han vuelto a observar rastros de tapir después de casi dos décadas de ausencia de esta especie en el territorio de la comunidad. Otro caso interesante fue el de las selvas de la cuenca alta del río Uxpanapa, donde se encontraron considerables extensiones de hábitat en excelente estado de conservación que albergan poblaciones de tapires, pecaríes de labios blancos, jaguares, pumas, monos y otros mamíferos grandes que han desaparecido desde hace ya décadas en la mayor parte de la cuenca baja de dicho río. Un tercer caso relevante es el del ejido El Pirú (Selva Lacandona), donde persisten poblaciones de ambas especies objeto del estudio. En esta comunidad los ejidatarios han decidido tomar medidas para restringir al mínimo la cacería y evitar deforestar las casi 3,000 hectáreas de selvas altas perennifolias que aún quedan en su territorio. Por último, los recientes registros arriba citados de tapir en Los Petenes y pecarí de labios blancos en el occidente de Campeche y el norte de Yucatán constituyen estímulos importantes para continuar y ampliar las investigaciones sobre la distribución y el estado de conservación de estas especies en el país.

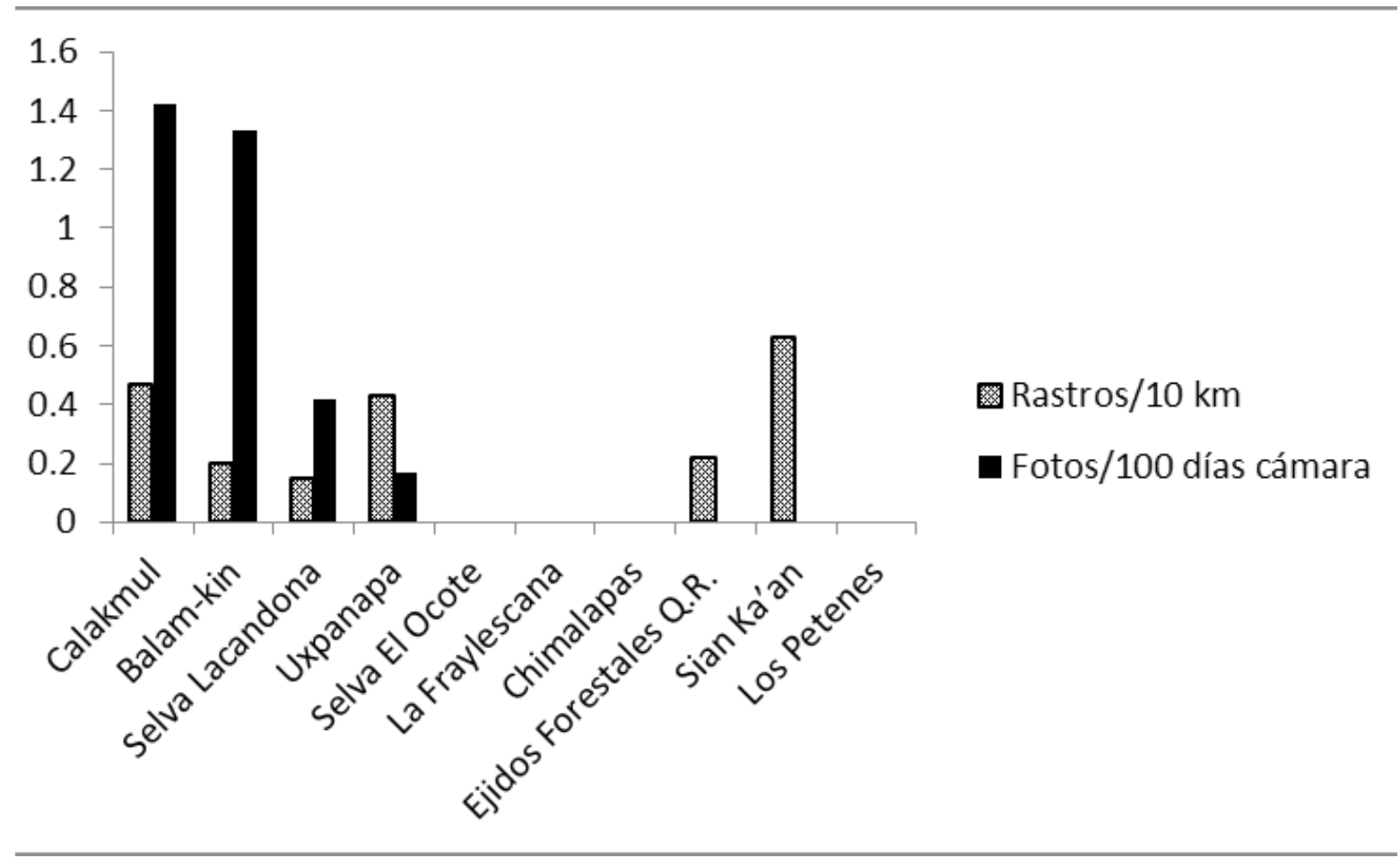

Figura 8. Abundancia del pecarí de labios blancos (Tayassu pecari) estimada mediante foto-trampeo y conteos de rastros en las localidades de estudio. 
Tabla 3. Índices de abundancia de tapir y pecarí de labios blancos obtenidos en transectos lineales y muestreos con cámaras-trampa en los sitios de estudio visitados entre noviembre 2010 y octubre 2012.

\begin{tabular}{|c|c|c|c|c|c|c|}
\hline \multirow[t]{2}{*}{ Sitio } & \multicolumn{3}{|c|}{ Tapirus bairdii } & \multicolumn{3}{|c|}{ Tayassu pecari } \\
\hline & $\begin{array}{c}\text { Registros } \\
\text { totales }\end{array}$ & $\begin{array}{l}\text { Rastros/ } \\
10 \mathrm{~km}\end{array}$ & $\begin{array}{c}\text { Fotografías/ } 100 \\
\text { días-cámara }\end{array}$ & $\begin{array}{c}\text { Registros } \\
\text { totales }\end{array}$ & $\begin{array}{c}\text { Rastros/ } \\
10 \mathrm{~km}\end{array}$ & $\begin{array}{c}\text { Fotografías/ } 100 \\
\text { días-cámara }\end{array}$ \\
\hline Selva Lacandona & 22 & 3.09 & 0.90 & 10 & 0.15 & 0.42 \\
\hline Selva El Ocote & 1 & 0.77 & 0 & 0 & 0 & 0 \\
\hline La Fraylescana & 7 & 4.38 & 0.53 & 0 & 0 & 0 \\
\hline Uxpanapa & 12 & 4.35 & 1.49 & 5 & 0.43 & 0.17 \\
\hline Chimalapas & 14 & 4.52 & 0.38 & 0 & 0 & 0 \\
\hline Ejidos Forestales Q. R. & 7 & 3.68 & 0.59 & 2 & 0.22 & 0 \\
\hline Sian Ka'an & 4 & 2.47 & 0 & 1 & 0.63 & 0 \\
\hline Calakmul & 6 & 3.30 & $2.00^{*}$ & 7 & 0.47 & $1.42^{*}$ \\
\hline Balam-kin & 5 & 2.11 & 0.89 & 1 & 0.20 & 1.33 \\
\hline Los Petenes & 1 & 0.26 & 0 & 0 & 0 & 0 \\
\hline
\end{tabular}

* Las cámaras se colocaron en aguadas.

\section{Conclusiones}

La información generada en el presente trabajo permitió mejorar el conocimiento acerca de la distribución actual, abundancia relativa y estado de conservación de las poblaciones de tapir y pecarí de labios blancos en México. Las áreas de distribución actuales y verificadas para el pecarí de labios blancos incluyen las Reservas de Calakmul, Balamkú, Balam-kin y Laguna de Términos (Campeche); las Reservas Lacantún y Montes Azules, la Comunidad Lacandona y algunos ejidos de Marqués de Comillas (Chiapas); Los Chimalapas (Oaxaca); Reserva Sian Ka'an y ejidos forestales del centro y sur (Quintana Roo), la cuenca alta del río Uxpanapa (Veracruz), y la Reserva de Dzilam (Yucatán). El tapir se encuentra en todas las anteriores a excepción de Dzilam, y además está presente en el Bajo Río Verde y partes bajas de las Sierras Mixe y Veinte Cerros (Oaxaca), Los Petenes (Campeche), y las Reservas El Ocote, El Triunfo, La Sepultura y algunos ejidos de la Sierra Madre (Chiapas). Por su gran extensión y buena calidad de hábitat, las áreas críticas para la conservación de ambas especies en el país son: Calakmul-Balamkú-Balam-kin, Montes Azules-Lacantún, Los Chimalapas, y Sian Ka'an-ejidos forestales del centro de Quintana Roo.

En este estudio se estimó con base en rastros y avistamientos directos una abundancia de 0.26 - 4.52 tapires, y 0.15 - 0.63 pecaríes de labios blancos por $10 \mathrm{~km}$ recorridos. Además se estimaron abundancias relativas de 0.38 - 1.49 (tapir) y 0.17 - 1.33 (pecarí) fotografías por 100 días-cámara. Estas cifras son las primeras estimadas mediante foto-trampeo para los dos ungulados en algunos de los sitios de estudio (Balam-kín, La Fraylescana y Uxpanapa), lo cual constituye una contribución significativa al estado del conocimiento sobre el estado de sus poblaciones. Las mayores abundancias de tapir se registraron en La Fraylescana, Uxpanapa y Los Chimalapas (rastros y avistamientos), y en Uxpanapa, Selva Lacandona y Balam-kin (fotografías). Las mayores abundancias de pecarí de labios blancos correspondieron a Sian Ka'an y Calakmul (rastros y avistamientos directos), y en Balam-kin y Calakmul (fotografías). 
Tabla 4. Principales amenazas detectadas para las poblaciones de tapir y pecarí de labios blancos en las comunidades visitadas en el sureste de México de marzo 2011 a octubre 2012.

\begin{tabular}{|c|c|c|c|c|}
\hline Sitio & $\begin{array}{c}\text { Amenazas } \\
\text { observadas } \\
\text { Tapir }\end{array}$ & $\begin{array}{l}\text { Riesgo* }^{*} \\
\text { Tapir }\end{array}$ & $\begin{array}{c}\text { Amenazas } \\
\text { observadas } \\
\text { Pecarí LB }\end{array}$ & $\begin{array}{l}\text { Riesgo* } \\
\text { Pecarí LB }\end{array}$ \\
\hline $\begin{array}{l}\text { Selva Lacandona, Subregión Marqués de Comillas } \\
\text { (Ejidos Adolfo López Mateos, Belisario } \\
\text { Domínguez, Benemérito de las Américas, Chajul, } \\
\text { Flor del Marqués, Galacia, José López Portillo, } \\
\text { Loma Bonita, Quiringuicharo, Playón de la Gloria, } \\
\text { Reforma Agraria y Zamora Pico de Oro). }\end{array}$ & DEF, CAZ, ENF & Medio & $\begin{array}{l}\text { CAZ, DEF, FRA, } \\
\text { ENF }\end{array}$ & Alto \\
\hline $\begin{array}{l}\text { Selva El Ocote } \\
\text { (Ejido Emilio Rabasa) }\end{array}$ & DEF, INC & Medio & Ausente & - \\
\hline $\begin{array}{l}\text { La Fraylescana } \\
\text { (Finca Arroyo Negro) }\end{array}$ & DEF, INC & Medio & Ausente & - \\
\hline $\begin{array}{l}\text { Uxpanapa } \\
\text { (Ejido Loma de Oro y Poblado El Quince) }\end{array}$ & $\begin{array}{l}\text { DEF, FRA, INC, CAZ, } \\
\text { ENF }\end{array}$ & Alto & $\begin{array}{l}\text { DEF, CAZ, FRA, } \\
\text { INC, ENF }\end{array}$ & Alto \\
\hline $\begin{array}{l}\text { Los Chimalapas } \\
\text { (Ejido La Fortaleza) }\end{array}$ & DEF, INC, CAZ, ENF & Alto & $\begin{array}{l}\text { CAZ, INC, DEF, } \\
\text { ENF }\end{array}$ & Alto \\
\hline Ejidos Forestales de Quintana Roo (Ejido Caobas) & INC, FRA & Medio & $\begin{array}{l}\text { CAZ, INC, FRA, } \\
\text { DEF }\end{array}$ & Alto \\
\hline $\begin{array}{l}\text { Sian Ka'an } \\
\text { (Ejidos X-Hazil y Chanká Veracruz) }\end{array}$ & INC, FRA & Medio & CAZ, INC, FRA & Medio \\
\hline $\begin{array}{l}\text { Calakmul } \\
\text { (Ejidos Nuevo Bécal y } 20 \text { de Noviembre) }\end{array}$ & INC & Bajo & CAZ, INC, ENF & Medio \\
\hline $\begin{array}{l}\text { Balam-kin } \\
\text { (Ejido Conhuas) }\end{array}$ & INC, CAZ & Medio & CAZ, INC & Medio \\
\hline $\begin{array}{l}\text { Los Petenes } \\
\text { (Ejido Zodzil) }\end{array}$ & DEF, FRA, INC, CAZ & Alto & Ausente & - \\
\hline
\end{tabular}

DEF: Deforestación; FRA: Fragmentación, CAZ: Caza; INC: Incendios; ENF: Enfermedades

* Escala de riesgo de acuerdo al número de factores de amenaza observados: Bajo (0-1); Medio (2-3); Alto (>3).

Las amenazas principales para la conservación del tapir y el pecarí de labios blancos en sus áreas de distribución actuales son la deforestación y fragmentación del hábitat, la cacería sin control, los incendios forestales, la sequía, las inundaciones y los huracanes. Algunos casos registrados de presencia inesperada de ambas especies en localidades puntuales de Campeche, Veracruz y Yucatán hacen evidente la necesidad de continuar y ampliar las investigaciones sobre la distribución y el estado de conservación de estos mamíferos en el sur y sureste del país.

Los resultados logrados en la presente investigación constituyen un avance significativo en el conocimiento acerca del estado de las poblaciones de tapir y pecarí de labios blancos en México. Se generó información actualizada sobre la distribución, abundancia y estado de conservación de diez poblaciones de tapir y seis de pecarí de labios blancos en los estados de Campeche, Chiapas, Oaxaca, Quintana Roo y Veracruz, aportando 105 registros de calidad al Sistema Nacional de Información sobre Biodiversidad (SNIB). A partir de las observaciones realizadas durante el estudio se identificaron los factores de amenaza más importantes para las poblaciones de las dos especies en el país. Esta información complementada con la revisión de literatura disponible, permitió elaborar propuestas para la ratificación de ambas especies en la NOM-059-SEMARNAT-2010 de acuerdo al Método de Evaluación de Riesgo (MER), así como proponer líneas de acción útiles para enriquecer las nuevas versiones de los Programas de Acción para la Conservación (PACE) de ambos mamíferos en México. 


\section{Agradecimientos}

Los autores agradecen a la CONABIO el apoyo financiero para llevar a cabo esta investigación (proyecto HK014), así como la información facilitada a través del Sistema Nacional de Información sobre Biodiversidad (SNIB). El Colegio de la Frontera Sur facilitó instalaciones, vehículos, equipo de campo y apoyo administrativo para la realización del proyecto. Numerosas personas otorgaron permisos y colaboraron de diversas maneras en el trabajo de campo en comunidades de Campeche (Ejidos Nuevo Becal, 20 de Noviembre, Conhuas y Zodzil), Chiapas (Ejidos Adolfo López Mateos, Belisario Domínguez, Benemérito de las Américas, Chajul, Flor del Marqués, Galacia, José López Portillo, Loma Bonita, Quiringuicharo, Playón de la Gloria, Reforma Agraria, Zamora Pico de Oro, Emilio Rabasa y Finca Arroyo Negro), Oaxaca (Ejido La Fortaleza), Quintana Roo (Ejidos X-Hazil, Chanká Veracruz y Caobas) y Veracruz (Ejido Loma de Oro y Poblado El Quince). La CONANP y el Gobierno de Campeche otorgaron amablemente permisos para realizar los muestreos en áreas protegidas de Campeche, Chiapas y Quintana Roo. A. Figueroa, L. Villaverde, E. Díaz, M. Sanvicente, R. Jiménez, N. Arias y N. Carrillo apoyaron durante parte del trabajo de campo del proyecto. Finalmente, los autores agradecen los oportunos comentarios de dos revisores anónimos para mejorar sustancialmente versiones previas del manuscrito.

\section{Resumen}

Introducción: El tapir centroamericano (Tapirus bairdii) y el pecarí de labios blancos (Tayassu pecari) juegan papeles importantes en la dinámica de los bosques tropicales en toda su área de distribución donde habitan a través de los procesos de herbivoría, dispersión y depredación de semillas. Estos mamíferos han constituido recursos alimentarios para los pobladores de comunidades rurales de Mesoamérica. Ambas especies aparecen en la NOM-059-SEMARNAT-2010, encontrándose en peligro de extinción debido principalmente a la pérdida de su hábitat y a la cacería sin control. El objetivo central del presente estudio consistió en generar un diagnóstico sobre el estado actual de las poblaciones de tapir y pecarí de labios blancos en México, proponiendo una estrategia para su conservación.

Métodos: De noviembre 2010 a noviembre 2012 se realizaron evaluaciones rápidas de la presencia, abundancia relativa y amenazas del tapir y el pecarí de labios blancos en diez localidades de cinco estados del sureste de México mediante tres técnicas complementarias: recorridos de transectos lineales, foto-trampeo y aplicación de entrevistas con pobladores locales. Con la información recabada y el análisis de la literatura disponible sobre ambas especies en México se generaron propuestas para confirmar su estatus de riesgo en la Norma Oficial Mexicana 059 mediante el Método de Evaluación de Riesgo (MER) requerido por la Secretaría de Medio Ambiente y Recursos Naturales (SEMARNAT).

Resultados y discussión: Las áreas de distribución actuales y verificadas para el pecarí de labios blancos se encuentran en los estados Campeche, Chiapas, Oaxaca, Quintana Roo, Veracruz y Yucatán. El tapir se encuentra en un mayor número de localidades dentro de los estados citados a excepción de Yucatán. Por su gran extensión y buena calidad de hábitat, las áreas críticas para la conservación de ambas especies en el país son: Calakmul-Balamkú-BalamKin (Campeche), Montes Azules-Lacantún (Chiapas), Los Chimalapas (Oaxaca), y Sian Ka'an-ejidos forestales del centro de Quintana Roo. Las mayores abundancias poblacionales estimadas de tapir se registraron en La Fraylescana, Uxpanapa y Los Chimalapas (rastros y avistamientos), y en Uxpanapa, Selva Lacandona y Balam-kin (fotografías). Las mayores abundancias estimadas de pecarí de labios blancos correspondieron a Sian Ka'an y Calakmul (rastros y avistamientos directos), y en Balam-kin y Calakmul (fotografías). Las amenazas principales para la conservación de ambas especies en México son la deforestación y fragmentación del hábitat, la cacería sin control, los incendios forestales y las enfermedades transmitidas por animales domésticos. Los registros inesperados de la presencia de ambas especies en localidades puntuales de Campeche, Veracruz y Yucatán estimulan la necesidad de continuar y ampliar las investigaciones sobre la distribución y el estado de conservación de estos mamíferos en el sur y sureste del país.

Palabras clave: amenazas, conservación, distribución, México, Tapirus bairdii, Tayassu pecari. 


\section{Literatura citada}

Arriaga, L., J.M. Espinoza, C. Aguilar, E. Martínez, L. Gómez, y E. Loa (coordinadores). 2000. Regiones terrestres prioritarias de México. Comisión Nacional para el Conocimiento y uso de la Biodiversidad. Ciudad de México, México. (http://www.conabio.gob.mx/conocimiento/regionalizacion/doctos/Tlistado.html)

Bodmer, R. E. 1991. Strategies of seed dispersal and seed predation in amazonian ungulates. Biotropica 23:255-261.

Bodmer, R., R. Aquino, P. Puertas, C. Reyes, T. Fang y N. Gottdenker. 1997. Manejo y Uso Sustentable de Pecaríes en la Amazonía Peruana. Comisión de Supervivencia de Especies UICN-Sur y CITES, Quito, Ecuador. Registros notables del tapir centroamericano (Tapirus bairdii) en la sierra Mixe. Oaxaca, México

Botello, F., O. Hernández, D. Reyes, V. Sánchez-Cordero y J. Sánchez. 2014. Registros notables del tapir centroamericano (Tapirus bairdii) en la Sierra Mixe, Oaxaca, México. Revista Mexicana de Biodiversidad 85:995-999.

Caughley, G. y A.R.E. Sinclair. 1994. Wildlife ecology and management. Blackwell Scientific. Oxford, Reino Unido.

Ceballos, G. 2008. Modelado de la distribución de las especies de mamíferos de México para un análisis GAP. Informe final SNIB-CONABIO proyecto DS006. EcoCiencia, S. C. Ciudad de México, México.

Comisión Nacional de Areas Naturales Protegidas (CONANP). 2009. Programa de Conservación de Especies en Riesgo (PROCER). http://www.conanp.gob.mx/especies.html.

Conroy, M. J. 1996. Abundance indices. Pp. 179-192 in Measuring and monitoring biological diversity: Standard methods for mammals (Wilson, D. E., F. R. Cole, J. D. Nichols, R. Rudran, y M. Foster, eds.). Smithsonian Institution Press. Washington, EE.UU.

CRUz, E. 2001. Hábitos de alimentación e impacto de la actividad humana sobre el tapir (Tapirus bairdii) en la Reserva de la Biosfera La Sepultura, Chiapas, México. Tesis de maestría. El Colegio de la Frontera Sur. San Cristóbal de Las Casas, México.

Cruz-Aldán, E., E. J. Naranjo, D. M. Güiris, P. Oropeza, E. Rendón, y L. Araujo. 2009. Programa de acción para la conservación de la especie (PACE): tapir centroamericano (Tapirus bairdii). Secretaría de Medio Ambiente y Recursos Naturales. Ciudad de México, México.

Cutler, T. L., Y D. E. Swann. 1999. Using remote photography in wildlife ecology: a review. Wildlife Society Bulletin 27:571-581.

Emmons, L. H., y F. Feer. 1997. Neotropical rainforest mammals. A field guide. Second edition. University of Chicago Press. Chicago, EE.UU.

Escamilla, A., M. Sanvicente, M. Sosa, y C. Galindo-Leal. 2000. Habitat mosaic, wildlife availability, and hunting in the tropical forest of Calakmul, México. Conservation Biology 14:1592-1601.

Escobedo, E. 2011. Mamíferos terrestres. Pp.267-271 in Riqueza Biológica de Quintana Roo. Un análisis para su conservación (Pozo, C., N. Armijo-Canto, y S. Calmé, eds.). El Colegio de la Frontera Sur (ECOSUR), Comisión Nacional para el Conocimiento y Uso de la Biodiversidad (Conabio), Gobierno del Estado de Quintana Roo y Programa de Pequeñas Donaciones. Ciudad de México, México.

Environmental Systems Research Institute (ESRI). 2008. ArcView 9. Redlands, CA, USA. http://www.esri.com/ software/arcgis/arcview/index.html.

Flesher, K. 1999. Preliminary notes on the conservation status of Baird's tapir in north-eastern Honduras. Oryx 33:294-300.

Gómez-Nísino, A. 2006. Ficha técnica de Tapirus bairdii. Los mamíferos mexicanos en riesgo de extinción según el PROY-NOM-059-ECOL-2000 (Medellín, R., compilador). Instituto de Ecología, Universidad Nacional Autónoma de México. Bases de datos SNIB-CONABIO. Proyecto No. W005. Ciudad de México, México.

Harmsen, B. J., R. J. Foster, S. Silver, L. Ostro, y C. P. Doncaster. 2010. Differential use of trails by forest mammals and the implications for camera-trap studies: A case study from Belize. Biotropica 42:126133. 
Instituto Nacional de Ecología (INE). 2000a. Programa de manejo, Reserva de la Biósfera Calakmul, México. Secretaría de Medio Ambiente, Recursos Naturales y Pesca. Ciudad de México, México.

Instituto Nacional de Ecología (INE). 2000b. Programa de manejo, Reserva de la Biósfera Montes Azules, México. Secretaría de Medio Ambiente, Recursos Naturales y Pesca. Ciudad de México, México.

Instituto Nacional de Ecología (INE). 2006. Programa de conservación y manejo, Reserva de la Biosfera Los Petenes. Secretaría de Medio Ambiente y Recursos Naturales. Ciudad de México, México.

International Union for Conservation of Nature and Natural Resources (IUCN). 2012. IUCN Red List of Threatened Species. Version 2012.2. <www.iucnredlist.org>. Accesado el 3 de noviembre 2012.

LiRA, I. 2006. Ecología y conservación del tapir centroamericano en la frontera de colonización de la zona noreste del municipio de Santa María Chimalapa, Oaxaca. Protocolo de tesis doctoral. Centro Interdisciplinario de Investigación para el Desarrollo Integral Regional (CIIDIR), Instituto Politécnico Nacional. Oaxaca, México.

LiRA, I., y M. Briones. 2011. Impacto de la ganadería extensiva y cacería de subsistencia sobre la abundancia relativa de mamíferos en la Selva Zoque, Oaxaca, México. Therya 2:217-244.

LiRA, I., M. Briones, y G. SÁnchez. 2014. Abundancia relativa, estructura poblacional, preferencia de hábitat y patrones de actividad del tapir centroamericano Tapirus bairdii (Perissodactyla: Tapiridae), en la Selva de Los Chimalapas, Oaxaca, México. Revista de Biología Tropical 62:1407-1419.

Lira, I., C. Galindo, y M. Briones. 2012. Mamíferos de la Selva Zoque: riqueza, uso y conservación. Revista de Biología Tropical 60:781-797.

LiRa, I., y E. J. Naranjo. 2005. Ampliación delárea de distribución de Tapirus bairdii, Gill 1865 (Perissodactyla: Tapiridae) en Oaxaca, México. Acta Zoológica Mexicana 21:107-110.

Lira, I., E. J. Naranjo, D. M. Güıris, y E. Cruz. 2004. Ecología de Tapirus bairdii (Perissodactyla:Tapiridae) en la Reserva de la Biósfera El Triunfo (Polígono I), Chiapas, México. Acta Zoológica Mexicana 20:1-21.

LiRa, I., Y E. J. Naranjo. 2005. Ampliación delárea de distribución de Tapirus bairdii, Gill 1865 (Perissodactyla: Tapiridae) en Oaxaca, México. Acta Zoológica Mexicana 21:107-110.

Lira, I., E. J. Naranjo, D. Hilliard, M. A. Camacho, A. De Villa y M. A. Reyes. 2006. Status and conservation of Baird's tapir in Oaxaca, Mexico. Tapir Conservation 15:21-28.

Loiselle, B. A., C. A. Howell, C. H. Graham, J. M., Goerck, T. Brooks, K. G. Smith, y P. H. Williams. 2003. Avoiding pitfalls of using species distribution models in conservation planning. Conservation Biology 17:1591-1600.

Lozano, R., y J. A. Olivares. 2011. Pp. 86-111 in Riqueza Biológica de Quintana Roo. Un análisis para su conservación (Pozo, C., N. Armijo-Canto y S. Calmé, eds.). El Colegio de la Frontera Sur (Ecosur), Comisión Nacional para el Conocimiento y Uso de la Biodiversidad (Conabio), Gobierno del Estado de Quintana Roo y Programa de Pequeñas Donaciones. Ciudad de México, México.

MARCH, I. J. 1990. Evaluación del hábitat y situación actual del pecarí de labios blancos (Tayassu pecari) en México. Tesis de maestría. Programa Regional en Manejo de Vida Silvestre, Universidad Nacional. Heredia, Costa Rica.

March, I. J. 1993. The white-lipped peccary (Tayassu pecary). Pp. 13-22 in Pigs, Peccaries and Hippos (Oliver, W. L. R., ed.). IUCN, Gland, Suiza.

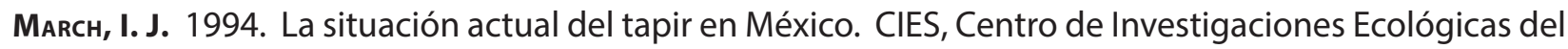
Sureste. Serie Monografías no.1. San Cristóbal de Las Casas, Chiapas, México.

March, I. J. 2005. Pecarí de labios blancos (Tayassu pecari). Pp. 522-524 in Los mamíferos silvestres de México (Ceballos, G. y G. Oliva, eds.). Comisión Nacional para el Conocimiento y Uso de la Biodiversidad, y Fondo de Cultura Económica. Ciudad de México, México.

March, I. J., y E. J. Naranjo. 2005. Tapir (Tapirus bairdii). Pp. 496-497 in Los mamíferos silvestres de México (Ceballos G. y G. Oliva, eds.). Comisión Nacional para el Conocimiento y Uso de la Biodiversidad y Fondo de Cultura Económica, México, D.F.

Mayer, J. J., y R. M. Wetzel. 1987. Tayassu pecari. Mammalian Species 293:1-7. 
Mendoza, E., T. Fuller, H. Thomassen, W. Buermann, D. Ramírez, y T. B. Smith. 2013. A preliminary assessment of the effectiveness of the Mesoamerican Biological Corridor

for protecting potential Baird's tapir (Tapirus bairdii) habitat in southern Mexico. Integrative Zoology 8:3547.

Muench, C. E. 2001. Patrones de uso del hábitat del tapir (Tapirus bairdii) en dos localidades de la selva lacandona, Chiapas. Tesis de licenciatura, Universidad Nacional Autónoma de México. Ciudad de México, México.

Naranjo, E. J. 1995. Abundancia y uso de hábitat del tapir (Tapirus bairdii) en un bosque tropical húmedo de Costa Rica. Vida Silvestre Neotropical 4:20-31.

Naranjo, E. J. 2000. Estimación de abundancia y densidad en poblaciones de fauna silvestre tropical. Pp. 37-46 in Manejo de Fauna Silvestre en Amazonia y Latinoamérica (Cabrera, E., C. Mercolli, y R. Resquín, eds.). Fundación Moisés Bertoni y CITES-Paraguay. Asunción, Paraguay.

Naranjo, E. J. 2002. Population ecology and conservation of ungulates in the Lacandon Forest, Mexico. Tesis doctoral, University of Florida. Gainesville, EE.UU.

Naranjo, E .J. 2009. Ecology and conservation of Baird's tapir in Mexico. Tropical Conservation Science 4:140-158.

Naranjo, E. J. 2012. Los incendios del 98 en la Selva Lacandona. Pp. 112-116 in Relatos de fogata: anécdotas y experiencias de biólogos y conservacionistas en campo (March I. J., y M. A. Lazcano, eds.). PNUD, CONANP y TNC. Ciudad de México, México.

Naranjo, E. J. 2014. Tapir. Pp. 377-387 in Ecología y manejo de fauna silvestre en México (Valdez, R., y J. A. Ortega, eds.). Colegio de Postgraduados y New Mexico State University. Texcoco, México.

Naranjo, E. J., S. A. Amador, F. Falconi, y R. Reyna. 2013. Conocimiento integral del tapir (Tapirus bairdii) y el pecarí de labios blancos (Tayassu pecari) en México. Informe técnico final del proyecto HK014. Comisión Nacional para el Conocimiento y Uso de la Biodiversidad. Ciudad de México, México.

Naranjo, E. J., y R. E. Bodmer. 2002. Population ecology and conservation of Baird's tapir (Tapirus bairdii) in the Lacandon Forest, Mexico. Tapir Conservation 11:25-33.

Naranjo, E. J., y R. E. Bodmer. 2007. Source-sink systems of hunted ungulates in the Lacandon Forest, Mexico. Biological Conservation 138:412-420.

Naranjo, E. J., J. E. Bolaños, M .M. Guerra, y R. E. Bodmer. 2004a. Hunting sustainability of ungulate populations in the Lacandon Forest, Mexico. Pp. 324-343 in People in nature: wildlife conservation in South and Central America (Silvius, K. M., R. E. Bodmer, y J. M. V. Fragoso, eds.). Columbia University Press. New York, EE.UU.

Naranjo, E. J., y E. Cruz. 1998. Ecología del tapir en la Reserva de la Biósfera La Sepultura. Acta Zoológica Mexicana 73:111-125.

Naranjo, E. J., M. M. Guerra, R. E. Bodmer, y J. E. Bolaños. 2004b. Subsistence hunting by three ethnic groups of the Lacandon Forest, Mexico. Journal of Ethnobiology 24:233-253.

Naranjo, E. J., y R. A. Reyna. 2007. Propuesta de inclusión del pecarí de labios blancos (Tayassu pecari) en el proyecto de modificación a la Norma Oficial Mexicana 059-ECOL-2001. Secretaría de Medio Ambiente y Recursos Naturales. Ciudad de México, México.

Nolasco, A. L. 2009. Distribución Actual y Estado de Conservación del Tapir Centroamericano Tapirus bairdii Gill, 1865 (Perissodactyla:Tapiridae) en México. Tesis de Licenciatura, Universidad Nacional Autónoma de México. Ciudad de México, México.o’Farrill, G., Calme, S., y Gonzalez, A. 2006. Manilkara zapota: A new record of a species dispersed by tapirs. Tapir Conservation 15:32-35.

Ojasti, J., y F. Dallmeier. 2000. Manejo de fauna silvestre Neotropical. Smithsonian Institution/Man and Biosphere Program. Washington, EE.UU.

Oımos, F. 1997. Tapirs as seed dispersers and predators. Pp. 3-9 in Tapir Action Plan (Brooks, D., R. E. Bodmer, y S. Matola, eds.). IUCN Tapir Specialists Group. Gland, Suiza. 
Ortega del Valle, D., T. Carranza, y J. Martínez. 2012. Una mirada desde el corazón de la Jícara de Oro (experiencias de conservación en la Selva Zoque de Los Chimalapas). World Wildlife Fund - México / USAID. Ciudad de México, México.

Pérez-Farrera, M. A., R. Martínez, E. Meléndez, E., Farrera, y H. Gómez. 2006. Inventario florístico de la Zona de Protección Forestal La Fraylescana, Chiapas, México. Informe final del proyecto Y012, CONABIO. Universidad de Ciencias y Artes de Chiapas. Tuxtla Gutiérrez, México.

Pérez, C., y E. Matus. 2010. El tapir Tapirus bairdii en la región sureste del Área de Protección de Flora y Fauna Balam Ka'ax, Quintana Roo, México. Therya 1:137-144.

Quisano, E. 2001. Ecología, aprovechamiento y conservación de la fauna silvestre en Tres Reyes, Quintana Roo. Tesis de Maestría, El Colegio de la Frontera Sur. Chetumal, México.

Reid, F. A. 1997. A field guide to the mammals of Central America and Southeast Mexico. Oxford University Press. New York, EE.UU.

Reyna. R. A. 2002. Hunting effects on the ungulates species in Calakmul Forest, Mexico. Tesis de maestría, University of Florida. Gainesville, EE.UU.

Reyna, R. A. 2007. Social ecology of the white-lipped peccary (Tayassu pecari) in Calakmul Forest, Campeche, Mexico. Tesis doctoral, University of Florida. Gainesville, EE.UU.

Reyna, R. A., I. J. March, E. J. Naranjo, y S. Mandujano. 2014. Pecaríes en México. Pp. 353-375 in Ecología y manejo de fauna silvestre en México (Valdez, R., y J. A. Ortega, eds.). Colegio de Postgraduados y New Mexico State University. Texcoco, México.

Reyna, R. A., E. J. Naranjo, C. A. Chapman, y G. W. Tanner. 2009. Hunting and the conservation of a social ungulate: The white-lipped peccary Tayassu pecari in Calakmul, Mexico. Oryx 44:89-96.

Reyna, R. A., y G. W. Tanner. 2005. Habitat preferences of ungulates in hunted and nonhunted areas in the Calakmul Forest, Campeche, Mexico. Biotropica 37:676-685.

Reyna-Hurtado, R., Y G. W. Tanner. 2007. Ungulate relative abundance in hunted and non-hunted sites in Calakmul Forest (Southern Mexico). Biodiversity and Conservation 16:743-757.

Rivadeneyra, E. 2007. Hábitos alimentarios del tapir (Tapirus bairdii) en el sector sur de la Reserva de la Biósfera Montes Azules, Chiapas, México. Tesis de licenciatura, Universidad Autónoma de Yucatán. Mérida, México.

Romero, S., B. G. Ferguson, D. Güiris, D. González, S. López, A. Paredes, y M. Weber. 2008. Comparative parasitology of wild and domestic ungulates in the Selva Lacandona, Chiapas, Mexico. Comparative Parasitology 75:115-126.

Secretaría de Desarrollo Social (Sedesol). 1993. Programa de manejo de la Reserva de la Biósfera de Sian Ka'an. Secretaría de Medio Ambiente, Recursos Naturales y Pesca. Ciudad de México, México.

Secretaría de Medio Ambiente y Recursos Naturales (SEMARNAT). 2001. Programa de manejo de la Reserva de la Biosfera Selva el Ocote. Comisión Nacional de Áreas Naturales Protegidas. Ocozocoautla, México.

Secretaría de Medio Ambiente y Recursos Naturales (SEMARNAT). 2010. Norma Oficial Mexicana NOM059-SEMARNAT-2010. Protección ambiental, especies nativas de flora y fauna silvestres de México, categorías de riesgo y especificaciones para su inclusión, exclusión o cambio, y lista de especies en riesgo. Diario Oficial de la Federación, 30 de diciembre de 2010:1-78.

Silveira, L. A.T.A., J.A. Jacomo, y F. Diniz-Filno. 2003. Camera trap, line transect census and track surveys: A comparative evaluation. Biological Conservation 114:351-355.

SowLs, L. K. 1997. Javelinas and the other peccaries: Their biology, management and use. Second ed. Texas A\&M University Press. College Station, EE.UU.

Tejeda-Cruz, C., E. J. Naranjo, A. D. Cuarón, H. Perales, y J. L. Cruz-Burguete. 2009. Habitat use of wild ungulates in fragmented landscapes of the Lacandon Forest, southern Mexico. Mammalia 73:211-219.

Vargas, F., y S. Escobar. 2000. Áreas naturales protegidas de México con decretos federales (1899-2000). Instituto Nacional de Ecología, SEMARNAP. Ciudad de México, México. 
Weber, M. 2000. Effects of hunting on tropical deer populations in Southeastern Mexico. Tesis de maestría, University of London. London, Reino Unido.

Submitted: October 23, 2014

Review: November 30, 2015

Accepted: January 26, 2015

Associated editor: Cristina MacSwiney 
TAPIR Y PECARÍ DE LABIOS BLANCOS EN MEXICO

250 THERYA Vol.6(1):227-249 